\title{
Spectroscopic properties of cool Ursa Major group members ${ }^{\star \star \star}$
}

\author{
M. Ammler-von Eiff ${ }^{1,2,3,4}$ and E. W. Guenther ${ }^{5}$ \\ ${ }^{1}$ Centro de Astronomia e Astrofísica da Universidade de Lisboa, Observatório Astronómico de Lisboa, Tapada da Ajuda, \\ 1349-018 Lisboa, Portugal \\ 2 Centro de Astrofísica da Universidade do Porto, Rua das Estrelas, 4150-762 Porto, Portugal \\ 3 Astophysikalisches Institut und Universitäts-Sternwarte Jena, Schillergäßchen 2-3, 07745 Jena, Germany \\ ${ }^{4}$ Georg-August-Universität Göttingen, Institut für Astrophysik, Friedrich-Hund-Platz 1, 37077 Göttingen, Germany \\ e-mail: mammler@uni-goettingen.de \\ 5 Thüringer Landessterwarte Tautenburg, 07778 Tautenburg, Germany \\ Received 18 June 2009 / Accepted 2 September 2009
}

ABSTRACT

\begin{abstract}
Context. Until now, most members of the Ursa Major (UMa) group of stars have been identified by means of kinematic criteria. However, in many cases kinematic criteria alone are insufficient to ascertain, whether an individual star is really a member of this group. Since photometric criteria are ineffective in the case of cool dwarf members, one must use spectroscopic criteria. Nevertheless, resulting membership criteria are inconclusive.

Aims. We reanalyse spectroscopic properties of cool UMa group dwarfs. In particular, we study the distribution of iron abundance, the strength of the Li I absorption at $6708 \AA$ and the Li abundance, and the infilling of the core of the $\mathrm{H} \alpha$ line.

Methods. Twenty-five cool and northern bona-fide members are carefully selected from the literature. Homogeneously measured stellar parameters and iron abundances are given for all Sun-like stars selected, based on spectra of high resolution and high signalto-noise ratio. In addition, we measure the $\mathrm{Li}$ equivalent width and abundance as well as the relative intensity of the $\mathrm{H} \alpha$ core and the corresponding chromospheric flux.

Results. The studied stars infer an average Ursa Major group iron abundance of $-0.03 \pm 0.05$ dex, which is higher by about 0.06 dex than determined elsewhere. The Li abundance derived of Ursa Major group dwarf stars is higher than in the Hyades at effective temperatures cooler than the Sun, but lower than in the younger Pleiades, a result which is independent of the exact value of the effective temperature adopted. The Sun-like and cooler dwarfs also display chromospheric infilling of the $\mathrm{H} \alpha$ core. We present spectroscopic criteria that may be used to exclude non-members.
\end{abstract}

Key words. stars: abundances - stars: fundamental parameters - open clusters and associations: individual: Ursa Major group solar neighborhood

\section{Introduction}

\subsection{The UMa group}

In the 19th century, Proctor (1869) and Huggins (1871) realized that five of the A stars in the Big Dipper constellation move to a common convergence point, and are thus likely to move into the same direction of space. In 1909, Hertzsprung identified stars at a very large angular distance from the Big Dipper constellation that were also moving towards the same convergent point, and thus had to be members of the same group of stars. All of these stars form an association with a central concentration, the UMa open cluster in the Big Dipper constellation, located at a distance of about $25 \mathrm{pc}$. Since the stars belonging to the UMa group probably do not form a single open cluster (Wielen 1978), the term Sirius supercluster has instead been suggested (Eggen 1994), or UMa association (Fuhrmann 2004), or UMa group. Since the nature of this cluster or association of stars is not yet well established, we prefer to use the more general term UMa group in this work. A number of reviews of the UMa group of stars have already been published: while Roman (1949)

^ Based on observations with the Coudé-Échelle spectrograph of the Alfred-Jensch-Teleskop at the Thüringer Landessternwarte Tautenburg.

$\star \star$ Based on observations collected at the Centro Astronómico Hispano Alemán (CAHA) at Calar Alto, operated jointly by the MaxPlanck-Institut für Astronomie and the Instituto de Astrofísica de Andalucía (CSIC). summarizes work until the middle of the 20th century, Eggen (1992) and Soderblom \& Mayor (1993b) review the preHipparcos era, and Asiain et al. (1999a) the post-Hipparcos research. Good accounts are also given by both King et al. (2003) and Fuhrmann (2004).

The UMa group of stars is well-suited to many different kinds of studies because of its relative proximity, which enabled the distances and proper motions of its members to be determined to high accuracy by the Hipparcos mission (ESA 1997; Perryman et al. 1997). The UMa group is young, its age being estimated to be $\approx 300 \mathrm{Myr}$ (von Hoerner 1957; Giannuzzi 1979; Duncan 1981; Soderblom \& Mayor 1993b) ${ }^{1}$. The young age of the UMa group is close to the time scale of the dissolution of open clusters (Wielen 1971). Studies of its members thus allow one to analyse aspects of stellar and cluster evolution.

\subsection{Kinematic and photometric membership criteria}

Is the lack of an established membership list the reason for the disagreement in the age of the UMa group?

\footnotetext{
1 The range of estimates varies significantly between 200 Myr (König et al. 2002) and $600 \mathrm{Myr}$ (King \& Schuler 2005). The age given by König et al. (2002) is based on the analysis of the Sun-like member $\chi^{1}$ Ori (HD 39587) and its low-mass companion while the photometric age given by King \& Schuler (2005) is constrained by the mainsequence turn-off of early-type members.
} 
The definition of kinematic membership criteria for the UMa group has always been a matter of debate. There are basically two different approaches. The first technique defines a list of canonical members, in this case the UMa nucleus, and derives kinematic criteria based on this sample of stars (e.g., Soderblom \& Mayor 1993b; Montes et al. 2001b; King et al. 2003). King et al. (2003) point out that the UMa group kinematics might be biased by the adoption of the canonical list of members. A second and unbiased approach looks for clustering in the kinematical space, an approach originating from Dziewulski (1916). Such work strongly benefits from the Hipparcos mission (e.g., Asiain et al. 1999a,b; Chereul et al. 1999).

In addition to kinematic criteria, photometric data can be used, since moving group members were born from the same material at approximately the same time. A photometric criterion was applied to the UMa group by King et al. (2003) and King \& Schuler (2005) who required that all members had to be located on the same isochrones throughout the HertzsprungRussell diagram implying an age of about $600 \mathrm{Myr}$. The method is effective for the early-type members of the UMa group since the isochrones are then sensitive to age. At cooler effective temperatures, i.e., in the case of solar and later type stars, luminosities and colours are less sensitive to age and the photometric criterion becomes less useful.

\subsection{Chemical homogeneity}

A similar abundance pattern is expected for members of moving groups since they are supposed to form from the same material. Indeed, observations show that stellar groups differ in terms of iron abundance, e.g., Boesgaard \& Friel (1990) measured $[\mathrm{Fe} / \mathrm{H}]=-0.03 \pm 0.02$ dex for the Pleiades, and $[\mathrm{Fe} / \mathrm{H}]=$ $0.13 \pm 0.05 \mathrm{dex}$ for the Hyades. For the UMa group, they inferred that $[\mathrm{Fe} / \mathrm{H}]=-0.085$, although a large spread of $0.20 \mathrm{dex}$ seems realistic (Soderblom \& Mayor 1993b; Gaidos \& Gonzalez 2002).

When abundance data are compiled from the literature, inhomogeneity is a major complication, as pointed out by King et al. (2003). The spread of abundance measurements for the same star can be very large as illustrated by the entry of the UMa group member HD 39587 in the catalogue of Cayrel de Strobel et al. (2001, Table 1). While the effective temperatures agree well, the surface gravities and abundances scatter widely. Therefore, an interesting question is how much of the iron abundance scatter detected in the UMa group is intrinsic.

\subsection{Spectroscopic indicators of youth}

The presence of Li I absorption at $\lambda \lambda 6707.8 \AA$ indicates stellar youth (e.g., Thorburn et al. 1993; Soderblom et al. 1993a; Carlsson et al. 1994). Young stars also display an enhanced level of activity, which can be spectroscopically observed as chromospheric infilling of the $\mathrm{H} \alpha$ line (Herbig 1985; Martin \& Montes 1997) or the Ca II H\&K lines, which has already been detected for the UMa group by Roman (1949).

A homogeneous level of both infilling of Balmer lines and $\mathrm{Li}$ absorption has also been detected (Montes et al. 2001a; Fuhrmann 2004; King \& Schuler 2005). Interestingly, Soderblom \& Mayor (1993b) noted that UMa group stars selected on the basis of spectroscopy also exhibit closer agreement with the kinematics of the nucleus of the UMa group. In the same fashion, King et al. (2003) point out that the dispersions in the space velocities increase from
Table 1. Inhomogeneity of literature data illustrated by the entry for the UMa group member HD 39587 in the catalogue of Cayrel de Strobel et al. (2001).

\begin{tabular}{llll}
\hline \hline$T_{\text {eff }}[\mathrm{K}]$ & $\log \left(g\left[\frac{\mathrm{cm}}{\mathrm{s}^{2}}\right]\right)$ & {$[\mathrm{Fe} / \mathrm{H}]$} & Source \\
\hline 5929 & & -0.05 & Boesgaard et al. (1988) \\
5929 & & -0.05 & Boesgaard (1989) \\
5929 & 4.50 & -0.05 & Boesgaard \& Friel (1990) \\
5900 & 4.21 & -0.05 & Friel \& Boesgaard (1992) \\
5953 & 4.46 & -0.03 & Edvardsson et al. (1993) \\
5895 & 4.21 & -0.04 & Gratton et al. (1996) \\
5950 & 4.46 & +0.11 & Mallik (1998) \\
$5929 \pm 70$ & $4.49 \pm 0.10$ & $-0.02 \pm 0.12$ & Castro et al. (1999) \\
$5805 \pm 70$ & $4.29 \pm 0.10$ & $-0.18 \pm 0.10$ & Chen et al. (2000) \\
\hline
\end{tabular}

those of the probable activity-based members to those of the activity-based non-members - a pattern that is also found for the UMa group sequence in the HR diagram.

Fuhrmann (2004) highlights that stars isolated from a complete sample of nearby stars based on spectroscopic features of activity, Li absorption, and enhanced projected rotational velocity have similar space velocities to those of the UMa cluster core.

\subsection{A new uniform study}

However, all this work is based either on only a small number of stars or on inhomogeneous data. So far, the definition of membership criteria beyond kinematics turns out to be difficult. King et al. (2003) realize
"... that activity is considerably more robust at excluding [UMa group] membership"

and

“... photometric membership and abundance-based membership are necessary but far from sufficient conditions to guarantee UMa membership."

Our new uniform work, which is based on as many late-type UMa group members as possible, will not only allow one to study the level of homogeneity of abundances, Li absorption, and $\mathrm{H} \alpha$ infilling. The new data may also help to refine previous approaches to defining spectroscopic membership criteria.

While the present work rectifies problems caused by inhomogeneous data or small sample numbers, it may still be affected by any problems related to the sample selection, which has to rely on a list of bona-fide members.

Table 2 compares the kinematic properties of the UMa group derived by different methods. We compare the most recent member lists, i.e, the lists published by Montes et al. (2001b, M01) and King et al. (2003, K03), which have 76 stars in common. Twenty-eight of these stars are kinematic members, according to M01, that fulfil both membership criteria. Sixteen of the 76 stars are kinematic members, according to $\mathrm{K} 03$, based on a different kinematic criterion ${ }^{2}$. Only 7 stars are assigned to be kinematic members by both M01 and K03 (see Fig. 1). The agreement between the kinematic membership lists is therefore poor, demonstrating that kinematic membership criteria alone are insufficient for determining whether a star is a member or not.

\footnotetext{
2 M01 use criteria according to Eggen (1958, 1995), which are not based on the individual components of the Galactic space motion in a similar way to $\mathrm{K} 03$, but on both the motion projected onto the celestial sphere and the total space velocity.
} 
Table 2. The UMa group kinematic parameters compiled from some recent studies.

\begin{tabular}{lccccll}
\hline \hline Reference & \multicolumn{2}{c}{ Convergent point } & \multicolumn{2}{c}{ Space velocity $\left[\mathrm{km} \mathrm{s}^{-1}\right]$} & Comments \\
& A (2000.0) & $\mathrm{D}(2000.0)$ & $U$ & $V$ & $W$ & \\
\hline Chereul et al. (1999) & & & $14.0 \pm 7.3$ & $1.0 \pm 6.4$ & $-7.8 \pm 5.5$ & scale 3 \\
Chupina et al. (2001) & 300.6864 & -29.74465 & $13.5 \pm 0.8$ & $3.0 \pm 0.9$ & $-7.5 \pm 2.0$ & UMa nucleus \\
Asiain et al. (1999a) & & & $8.7 \pm 6.6$ & $2.8 \pm 4.1$ & $-6.9 \pm 5.8$ & group A \\
M01 & 20.55 & -38.10 & 14.9 & 1.0 & -10.7 & \\
K03 & & & $13.9 \pm 0.6$ & $2.9 \pm 0.9$ & $-8.4 \pm 1.3$ & UMa nucleus, weighted \\
\hline
\end{tabular}

The comments in the last column specify details regarding the given literature source.
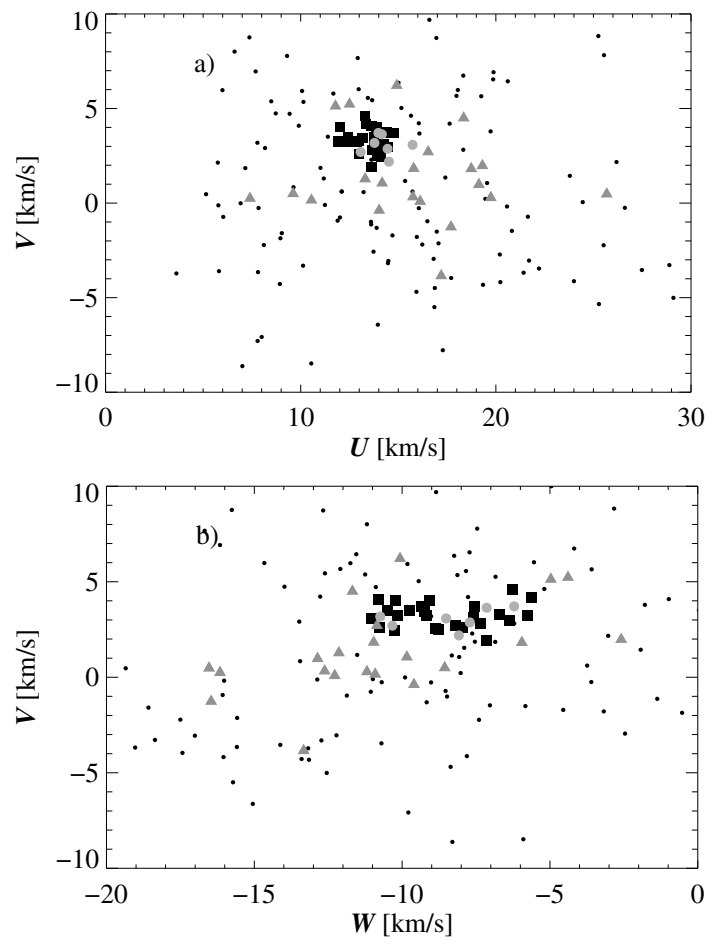

Fig. 1. Space velocities of the kinematic sample - a) The Galactic space velocities $U$ and $V$ of kinematic members and further UMa group candidates are displayed. Triangles indicate the stars satisfying both membership criteria of M01. Squares denote the stars that agree with the kinematic criterion of K03. Circles are the seven stars that fulfil the criteria of both M01 and K03. Dots are additional UMa group candidates taken from literature. b) Same as a), now for $W$ and $V$. Note that the dots do not fulfil both $U V$ and $W V$ constraints simultaneously. If space velocities are given by both M01 and K03, then they are taken from the former.

The selection of the most recent membership list (K03) was based on a combination of kinematic, photometric, and spectroscopic membership criteria. Out of 220 candidates, nearly 60 assured members are presented, although only the selection of 30 of these is consistent with the applied kinematic criteria. We study the homogeneity of spectroscopic UMa group features, so our conclusions are biased when using the final member list of $\mathrm{K} 03$ as it is.

Presently, the best solution seems to be to study different lists of members instead of trying to define a new ultimate list. Therefore, spectroscopic properties are considered separately for the list of kinematic members (lists $M 01 \mathrm{k}$ and $K 03 \mathrm{k}$ ) and for the list of final members of K03 (list K03f):

- M01k - 28 kinematic members according to M01 (fulfilling both of Eggen's criteria);
- K03k-36 kinematic members according to K03 (assignment $Y)$;
- K03f - 31 final members according to K03 (assignment $Y$ without question mark).

From these (overlapping) lists of in total 58 stars, the 25 lowmass northern members ${ }^{3}$ are the subject to the present work (Table 3).

Iron abundances are presented for the 17 Sun-like stars, six of which already were analysed with the same methods. For these stars and eight more, i.e., in total 25 late-type stars, Li absorption and chromospheric infilling of $\mathrm{H} \alpha$ is addressed.

Table 3 lists stellar data relevant to this work. As in F04, $V$ band magnitudes are derived from Hipparcos photometry using the conversion of Bessell (2000). In the case of the five stars analysed by F04, the slightly different values given by F04 were adopted for consistency reasons.

\section{Observations and data reduction}

The spectra were obtained in 2002-2004 with the high resolution Échelle spectrograph FOCES at the $2.2 \mathrm{~m}$-telescope of the Centro Astronómico Hispano Alemán (CAHA) at Calar Alto (Pfeiffer et al. 1998; Grupp 2003, 2004), and the CoudéÉchelle spectrograph at the $2 \mathrm{~m}$-Alfred-Jensch telescope of the Thüringer Landessternwarte at Tautenburg (Hatzes et al. 2005; König 2003). Since the analysis works differentially with respect to the Sun, we also obtained solar spectra by observing the illuminated disk of the Moon during each observing campaign ${ }^{4}$.

Some of the stars were already analysed by F04, although no measurement of either the Li equivalent width or the chromospheric flux was made. Spectra of these stars observed in the same way with FOCES as in the present work were kindly provided by K. Fuhrmann for this purpose.

The FOCES setup included a slit width of $120 \mu \mathrm{m}$ and the use of the Loral $11 \mathrm{i} 2 \mathrm{k} \times 2 \mathrm{k}$ chip. Spectra with a resolution of $\lambda / \Delta \lambda \gtrsim 60000$ were obtained. The TLS Échelle observations were characterized by a slit width of $520 \mu \mathrm{m}$ and the use of the $2 \mathrm{k} \times 2 \mathrm{k}$ EEV chip with $13.5 \times 13.5 \mu \mathrm{m}$ pixels achieving a resolution of $\lambda / \Delta \lambda=67000$. A signal-to-noise ratio of at least 200 close to $\mathrm{H} \alpha$ was obtained for most spectra.

For our analyses, we used the wavelength region from 4700 to $7400 \AA$ in the case of the TLS Échelle spectrograph, and

\footnotetext{
3 The 21 early-type stars in the final list are not studied in this work. Furthermore, five stars on the southern hemisphere are omitted since our observations were restricted to the northern hemisphere. Five more stars are close companions of brighter members and could not be resolved with the spectrographs.

4 The observations of HD 109011 are the only case that solar spectra have not been obtained during the same campaign.
} 
Table 3. The sample of UMa group members.

\begin{tabular}{|c|c|c|c|c|c|c|c|c|c|c|c|c|}
\hline \multirow[t]{2}{*}{ Object } & \multirow[t]{2}{*}{ Spectral type } & \multirow[t]{2}{*}{$V$} & \multirow{2}{*}{$\begin{array}{c}\pi \\
{[\mathrm{mas}]}\end{array}$} & \multicolumn{2}{|c|}{ M01 } & \multicolumn{3}{|c|}{ K03 } & \multirow[t]{2}{*}{ F04 } & \multicolumn{3}{|c|}{ Sub-sample } \\
\hline & & & & pec. vel. & rad. vel. & kin. & phot. & final & & & & \\
\hline HD 11131 & G1V & $6.727 \pm 0.005^{1}$ & $42.35 \pm 0.87^{3}$ & $\mathrm{Y}$ & $\mathrm{Y}$ & $?$ & Y? & Y? & $\mathrm{Y}$ & M01k & & \\
\hline HD 24916 A & K4V & $8.033 \pm 0.018$ & $63.41 \pm 2.00$ & $\mathrm{Y}$ & Y & $\mathrm{N}$ ? & $?$ & $\mathrm{~N}$ ? & & M01k & & \\
\hline HD 26923 & G0IV & $6.314 \pm 0.005^{1}$ & $47.20 \pm 1.08$ & Y & Y & Y? & Y & Y? & Y & M01k & & \\
\hline HD 28495 & G0V & $7.748 \pm 0.023$ & $36.32 \pm 1.07$ & $\mathrm{~N}$ & $\mathrm{Y}$ & $\mathrm{Y}$ & $\mathrm{Y}$ & $\mathrm{Y}$ & & & K03k & $K 03 f$ \\
\hline HD 38393 & F7V & $3.586 \pm 0.0044$ & $111.49 \pm 0.60$ & Y & Y & $? / \mathrm{Y} ?$ & $\mathrm{Y}$ ? & Y? & & M01k & & \\
\hline HD 39587 & G0V & $4.396 \pm 0.005^{1}$ & $115.43 \pm 1.08$ & & & Y & $\mathrm{Y}$ & $\mathrm{Y}$ & $\mathrm{Y}$ & & K03k & $K 03 f$ \\
\hline HD 41593 & K0V & $6.762 \pm 0.005^{1}$ & $64.71 \pm 0.91$ & $\mathrm{Y}$ & $\mathrm{Y}$ & $\mathrm{N} ? / ?$ & $\mathrm{Y}$ & $\mathrm{N} ? / ?$ & Y & M01k & & \\
\hline HD 59747 & G5V & $7.677 \pm 0.005^{1}$ & $50.80 \pm 1.29$ & Y & Y & Y & $\mathrm{Y} ?$ & Y & $\mathrm{Y}$ & M01k & K03k & $K 03 f$ \\
\hline HD 71974 A & G5V & $7.265 \pm 0.011$ & $34.83 \pm 1.37$ & $\mathrm{Y}$ & $\mathrm{N}$ & $\mathrm{Y}$ & Y? & Y & & & $K 03 k$ & KO3f \\
\hline HD 95650 & M0 & $9.508 \pm 0.024$ & $85.76 \pm 1.36$ & $\mathrm{~N}$ & $\mathrm{Y}$ & Y & Y? & Y & & & K03k & K03f \\
\hline HD 238087 & K5 & $9.975 \pm 0.030$ & $35.24 \pm 1.24$ & $\mathrm{Y}$ & $\mathrm{Y}$ & Y? & $?$ & $?$ & & M01k & & \\
\hline HD 109011 & $\mathrm{~K} 2 \mathrm{~V}$ & $8.069 \pm 0.015$ & $42.13 \pm 3.11$ & & & $\mathrm{Y}$ & Y & Y & & & K03k & $K 03 f$ \\
\hline HD 109647 & K0V & $8.503 \pm 0.020$ & $38.08 \pm 1.11$ & $\mathrm{Y}$ & $\mathrm{Y}$ & Y & Y & Y & & M01k & $K 03 k$ & $K 03 f$ \\
\hline HD 110463 & K3V & $8.256 \pm 0.018$ & $43.06 \pm 0.82$ & Y & Y & $\mathrm{Y}$ & Y & $\mathrm{Y}$ & & M01k & K03k & $K 03 f$ \\
\hline HD 112196 & F8V & $6.985 \pm 0.015$ & $29.19 \pm 1.60$ & $\mathrm{Y}$ & Y & $\mathrm{N} ? / ?$ & $?$ & $\mathrm{~N} ? / ?$ & & M01k & & \\
\hline HD 115043 & G1Va & $6.814 \pm 0.011$ & $38.92 \pm 0.67$ & $\mathrm{Y}$ & Y & $\mathrm{Y}$ & $\mathrm{Y}$ & $\mathrm{Y}$ & & M01k & K03k & $K 03 f$ \\
\hline HD 238224 & K5 & $9.642 \pm 0.022$ & $39.84 \pm 1.44$ & Y & Y & Y & ? & Y? & & MO1k & K03k & \\
\hline HD 152863 A & G5III & $6.066 \pm 0.008$ & $7.14 \pm 0.67$ & $\mathrm{Y}$ & $\mathrm{Y}$ & $?$ & Y? & $?$ & & M01k & & \\
\hline HD 155674 A & K0 & $8.903 \pm 0.087$ & $47.14 \pm 1.88$ & Y & Y & $?$ & $?$ & $?$ & & M01k & & \\
\hline HD 155674 B & K8 & $9.304 \pm 0.054$ & $47.86 \pm 3.11$ & $\mathrm{Y}$ & $\mathrm{Y}$ & ? & ? & $?$ & & M01k & & \\
\hline HD 167389 & F8 & $7.383 \pm 0.010$ & $29.91 \pm 0.59$ & Y & Y & $\mathrm{N}$ ? & $?$ & $\mathrm{~N} ? / ?$ & & M01k & & \\
\hline HD 171746 A & G2V & $6.924 \pm 0.071^{2}$ & $29.23 \pm 1.54$ & $\mathrm{~N}$ & $\mathrm{Y}$ & $\mathrm{Y}$ & $\mathrm{Y} ?$ & $\mathrm{Y}$ & & & K03k & $K 03 f$ \\
\hline HD 171746 B & $\mathrm{G} 2 \mathrm{~V}$ & $7.009 \pm 0.071^{2}$ & $29.23 \pm 1.54$ & & & $\mathrm{Y}$ & Y? & $\mathrm{Y}$ & & & K03k & $K 03 f$ \\
\hline HD 184960 & F7V & $5.714 \pm 0.006$ & $39.08 \pm 0.47$ & $\mathrm{Y}$ & $\mathrm{Y}$ & $? / \mathrm{Y} ?$ & $?$ & $? / \mathrm{Y} ?$ & & M01k & & \\
\hline HD 205435 & G8III & $3.986 \pm 0.006$ & $26.20 \pm 0.51$ & $\mathrm{Y}$ & $\mathrm{Y}$ & $? / \mathrm{Y} ?$ & ? & $?$ & & M01k & & \\
\hline
\end{tabular}

The table gives the spectral type (2), the $V$ band magnitude (3) derived from the Hipparcos system using the conversion of Bessell (2000) in most cases, the parallax $\pi(4)$, Eggen's peculiar velocity criterion (5) and Eggen's cluster radial velocity criterion (6) as applied by M01, the kinematic $U V W$ criterion (7), photometric criterion (8), and final membership according to K03 (9), and the spectroscopic membership following F04 (10). The last column indicates the membership of the sub-samples $M 01 k, K 03 k$, and $K 03 f$ as described in the text in detail. Most astrometric and photometric measurements are taken from the Hipparcos catalogue (Perryman et al. 1997). Exceptions are indicated in the footnote below.

${ }^{1}$ F04. ${ }^{2}$ Based on values in the Hipparcos system given by Fabricius \& Makarov (2000). See Sect. 3.3 for more details. ${ }^{3}$ According to F04, the parallax of the primary HD 11171 is adopted.

from 4100 to $8200 \AA$ in the case of FOCES. Both spectrographs cover simultaneously all relevant $\mathrm{Fe} I$ and FeII-lines ${ }^{5}$ (Table A.1), as well as $\mathrm{H} \alpha, \mathrm{H} \beta$, the $\mathrm{Mg} \mathrm{Ib}$ triplet, and the Li 6707.8 A doublet (Table 4).

Using the FOCES EDRS data reduction package that Klaus Fuhrmann adapted for use with the TLS Échelle, we followed the usual steps of data reduction (Horne 1986; McLean 1997): bias subtraction, removal of scattered light, simple order extraction, wavelength calibration using Th-Ar exposures, flat-field division, rectification, and merging of all Échelle orders into one spectrum.

The accurate normalization of the relative continuum - often called "rectification" - is very important in the region of the Balmer lines, whose wings are required for the determination of the effective temperatures. The problem is that chromospheric lines such as $\mathrm{H} \alpha$ are so broad that the wings of these lines extend over more than one Échelle-order. The normalization of the FOCES spectra is straightforward and completed by using spectra taken with the flat-field lamp, because the illumination of either a lamp or a star is identical in this fibre-fed spectrograph (see Korn 2002, for a related discussion). In Tautenburg, spectra of a

\footnotetext{
5 Not all lines can be used for each star. The presence of blends as well as the equivalent width of individual lines may vary strongly between the stars. Only lines with equivalent widths lower than some $80 \mathrm{~m} \AA$ may be used since the UMa group is young and stronger lines might be affected by chromospheric effects. Usually, $30 \mathrm{Fe} \mathrm{I}$ and $10 \mathrm{Fe} \mathrm{II} \mathrm{lines}$ out of the full list can be used for an individual star.
}

Table 4. Mg I and Li I line data.

\begin{tabular}{|c|c|c|c|c|c|}
\hline $\begin{array}{c}\lambda \\
{[\AA]}\end{array}$ & $\begin{array}{l}\chi_{\text {low }} \\
{[\mathrm{eV}]}\end{array}$ & $\log g f$ & $\log C_{6}$ & $\begin{array}{c}\gamma_{\mathrm{rad}} \\
10^{8}[1 / \mathrm{s}]\end{array}$ & $\log C_{4}$ \\
\hline \multicolumn{6}{|c|}{ Mg I } \\
\hline 4571.097 & 0.00 & -5.59 & -31.50 & - & -15.00 \\
\hline 4730.031 & 4.33 & -2.34 & -28.80 & 4.768 & -15.00 \\
\hline 5167.336 & 2.70 & -0.87 & -30.88 & 1.002 & -14.52 \\
\hline 5172.697 & 2.70 & -0.39 & -30.88 & 1.002 & -14.52 \\
\hline 5183.616 & 2.70 & -0.17 & -30.88 & 1.002 & -14.52 \\
\hline 5528.415 & 4.33 & -0.50 & -30.43 & 4.908 & -13.12 \\
\hline 5711.093 & 4.33 & -1.70 & -30.00 & 4.817 & -15.00 \\
\hline 5785.280 & 5.11 & -1.76 & -30.20 & - & -15.00 \\
\hline \multicolumn{6}{|c|}{ Li I } \\
\hline 6707.760 & 0.00 & 0.00 & -31.86 & - & -15.00 \\
\hline 6707.910 & 0.00 & -0.31 & -31.86 & - & -15.00 \\
\hline
\end{tabular}

Following values are listed: (1) central wavelength, (2) lower excitation potential, (3) oscillator strength, (4) van der Waals damping constant, (5) radiative damping constant, (6) quadratic Stark effect damping constant. The radiation damping constant $\left(\gamma_{\mathrm{rad}}\right)$ is calculated with the classical formula within LINFOR in case it is missing in the table.

standard flat-field lamp placed in front of the slit was found to be unsuitable for the normalization. Thus, a new flat-field device was installed, where four $500 \mathrm{~W}$ continuum lamps illuminate homogeneously a white screen in the dome at which the telescope is pointed. The light from the lamp is then reflected in the same way as starlight before entering the slit of the spectrograph. This 
is the case for all mirrors of the telescope including the Coudémirror train. The flat-field device was tested thoroughly by observing many stars, including $\alpha \mathrm{Lyr}$, and found to be capable for normalizing well the spectra.

\section{Stellar parameters and iron abundance}

\subsection{Methods}

To derive the stellar parameters, model atmospheres in local thermodynamic equilibrium (LTE) were used. These comprise temperature and pressure profiles computed with MAFAGS, an unpublished code originally developed by T. Gehren and later revised by Reile (1987). Spectral profiles are calculated using the unpublished line formation code LINFOR, which is based on MAFAGS and was developed by T. Gehren, C. Reile, K. Fuhrmann, and J. Reetz.

The model atmospheres are based on the plane-parallel approximation and hydrostatic equilibrium is assumed. The elemental abundance pattern is based mostly on Holweger (1979). Line opacity is accounted for by scaled opacity distribution functions (Kurucz 1995). Convection is accounted for by the mixing-length approximation. LINFOR reads the temperature and pressure profiles and solves the radiative transfer in LTE for hydrogen, neutral, and singly ionized iron as well as neutral magnesium. For more details, the reader is referred to Fuhrmann (1993) and Fuhrmann et al. (1997a). Additional information about MAFAGS and LINFOR is given in both Ammler (2006a,b) and Korn et al. (2005).

Since Fuhrmann's method for deriving the stellar parameters was extensively described in the literature (Fuhrmann 1993; Fuhrmann et al. 1993, 1997a; Fuhrmann 1998; Ammler 2006a,b), only the basic ideas are summarized briefly here. Effective temperature, surface gravity, microturbulence, and iron abundance are determined iteratively in several steps. Effective temperature is derived from the Balmer line wings, and the surface gravity is determined from either the wings of the $\mathrm{Mg} \mathrm{Ib}$ triplet or the ionization equilibrium of iron (Fe I and Fe II). The iron abundance, the microturbulence parameter, and the projected rotational velocity are determined from the profiles of unblended Fe II lines.

\subsubsection{Effective temperature}

Effective temperature is derived by fitting synthetic line profiles to the wings of the $\mathrm{H} \alpha^{6}$ line according to Fuhrmann et al. (1994, and previous publications). One minor complication is that the UMa group is so young that the core of $\mathrm{H} \alpha$ is partly filled-in by chromospheric emission. To estimate the effect of the chromospheric infilling, temperature is assessed additionally by fitting the wings of $\mathrm{H} \beta$, which is less affected by chromospheric activity (also see Fuhrmann 2004). Both values agree well leading to the conclusion that the infilling mostly affects the cores, not the wings of the lines. Only in the case of HD 28495 are there residuals of the fits in the inner part of the $\mathrm{H} \alpha$ wings that may be due to activity (see discussion of individual objects in Sect. 3.3). This object displays an elevated level of chromospheric flux (see Table 6 and Figs. 8 and 9).

An error bar is estimated from the noise, by fitting upper and lower envelopes to the noise features. These estimates are usually below $100 \mathrm{~K}$.

\footnotetext{
${ }^{6}$ The $\mathrm{H} \beta$ and $\mathrm{H} \gamma$ lines are strongly blended in all of the studied spectra and do not allow one to derive effective temperature. $\mathrm{H} \beta$ can still be used to check the consistency of the temperatures derived with $\mathrm{H} \alpha$.
}

\subsubsection{Surface gravity from the iron ionization equilibrium}

Surface gravity is derived either from the iron ionization equilibrium, or from the wings of the $\mathrm{Mg} \mathrm{Ib}$ triplet, or from the Hipparcos distance if both spectroscopic methods fail.

For all stars with temperatures between 5000 and $6000 \mathrm{~K}$ (HD 28495, HD 109647, HD 110463, HD 115043), the iron ionization balance was imposed to derive the surface gravity. This method requires the measurement of the equivalent widths of about 30 unblended Fe I lines and 10 of Fe II. These are inspected and selected individually for each spectrum from a list of more than 100 lines (see Table A.1).

The derived surface gravity varies strongly when changing the adopted effective temperature within its error bars. Furthermore, the result depends on the adopted iron abundance and the value of the microturbulence parameter ${ }^{7}$. Therefore, surface gravity is determined iteratively together with iron abundance and the microturbulence parameters (both from Fe II lines only), i.e., all of these parameters are determined consistently from the spectrum.

Error bars for all these values are estimated by varying the effective temperature within its error bars. The resulting error bar of surface gravity is typically smaller than 0.15 dex. These values may underestimate the true errors involved. Since all possible systematic errors cannot be practically taken into account, the reliability of the surface gravities is assessed by various tests (Sect. 3.2).

\subsubsection{Surface gravity from strong lines}

For stars with a temperature of about $5900 \mathrm{~K}$ or more, the application of the ionization equilibrium may underestimate the surface gravity (i.e., for HD 167389, HD 184960). The problem seems to arise from the Fe I lines. In the case of the warmer stars, most iron is ionized and the Fe I lines become strongly dependent on the details of the temperature structure, which might be insufficiently predicted by the LTE model atmospheres (cf. Fuhrmann et al. 1997a). In these cases, surface gravity is assessed by fitting the wings of the $\mathrm{Mg} \mathrm{Ib}$ triplet ${ }^{8}$. The best results are obtained with Mg I $\lambda 5172 \AA$ and $\lambda 5183 \AA$. An estimate of $\mathrm{Mg}$ abundance is needed and is obtained from weak Mg I lines (e.g., $\lambda 4571 \AA$ and $\lambda 5711 \AA$, see Table 4). In the case of HD 167389, the fit is complicated and may produce a slightly spurious surface gravity (see discussion of individual objects in Sect. 3.3).

The error bars are inferred in the same way as before (Sect. 3.1.2) but now the iteration also incorporates the derivation of the $\mathrm{Mg}$ abundance. The resulting error bars are roughly the same as in the use of the iron ionization balance (also see Fuhrmann et al. 1997a).

\subsubsection{Surface gravity from Hipparcos parallaxes}

For some stars hotter than $\approx 5900 \mathrm{~K}$, we found that even the use of the magnesium wings fails (HD 38393, HD 112196, HD 171746A, and HD 171746B), and therefore, surface

\footnotetext{
7 Except in the case of very weak lines, the adoption of a wrong microturbulence parameter causes spurious abundance determinations. To have a sufficient number of lines at our disposal, we need to include all useful lines with equivalent widths $<80 \mathrm{~m} \AA$ and therefore have to account properly for microturbulence.

8 For a discussion of the preference of magnesium with respect to other strong lines, we refer to Fuhrmann et al. (1997a).
} 
Table 5. Stellar parameters of the 18 Sun-like stars.

\begin{tabular}{|c|c|c|c|c|c|c|c|c|c|c|c|c|c|c|}
\hline Object & $\begin{array}{l}T_{\text {eff }} \\
{[\mathrm{K}]}\end{array}$ & $\begin{array}{r}\log g \\
{\left[\mathrm{~cm} / \mathrm{s}^{2}\right]} \\
\end{array}$ & {$[\mathrm{Fe} / \mathrm{H}]$} & {$[\mathrm{Mg} / \mathrm{Fe}]$} & $\begin{array}{c}\xi_{\mathrm{t}} \\
{\left[\mathrm{km} \mathrm{s}^{-1}\right]}\end{array}$ & $\begin{array}{c}\zeta_{\mathrm{RT}} \\
{\left[\mathrm{km} \mathrm{s}^{-1}\right]}\end{array}$ & $\begin{array}{c}v \sin i \\
{\left[\mathrm{~km} \mathrm{~s}^{-1}\right]}\end{array}$ & ref. & $\begin{array}{c}M \\
{\left[M_{\odot}\right]}\end{array}$ & $\begin{array}{c}R \\
{\left[R_{\odot}\right]} \\
\end{array}$ & B.C.V & $M_{\text {bol }}$ & $\begin{array}{r}d_{\mathrm{sp}} \\
{[\mathrm{pc}]}\end{array}$ & $\begin{array}{l}\frac{d_{\mathrm{sp}}-d_{\mathrm{HIP}}}{d_{\mathrm{HIP}}} \\
{[\%]}\end{array}$ \\
\hline \multicolumn{15}{|l|}{$M 01 k$} \\
\hline \multirow[t]{2}{*}{ HD 11131} & 5796 & 4.43 & -0.10 & 0.00 & 0.97 & 3.70 & 1.5 & 2 & 1.00 & 1.01 & -0.13 & 4.73 & 23.8 & 0.6 \\
\hline & 70 & 0.10 & 0.07 & 0.05 & 0.20 & & 1.0 & & 0.05 & 0.04 & & 0.07 & 3.3 & \\
\hline \multirow[t]{2}{*}{ HD 24916 A } & 4600 & 4.60 & -0.03 & - & - & - & 2.1 & 3 & 0.70 & 0.69 & -0.57 & 6.48 & 15.4 & -2.4 \\
\hline & 100 & 0.20 & 0.15 & - & - & & 2.0 & & 0.05 & 0.03 & & 0.09 & 3.7 & \\
\hline \multirow[t]{2}{*}{ HD 26923} & 5975 & 4.41 & -0.06 & -0.01 & 0.99 & 4.40 & $<1.0$ & 2 & 1.07 & 1.01 & -0.11 & 4.57 & 22.4 & 5.5 \\
\hline & 70 & 0.10 & 0.07 & 0.05 & 0.20 & & - & & 0.05 & 0.04 & & 0.07 & 3.0 & \\
\hline \multirow[t]{2}{*}{ HD 38393} & 6310 & $4.28^{1}$ & -0.05 & 0.01 & 1.30 & 5.70 & 8.1 & 1 & 1.23 & 1.33 & -0.08 & 3.74 & - & - \\
\hline & 60 & 0.03 & 0.05 & 0.06 & 0.20 & & 1.0 & & 0.05 & 0.04 & & 0.05 & - & \\
\hline \multirow[t]{2}{*}{ HD 41593} & 5278 & 4.54 & 0.02 & -0.01 & 1.12 & 1.70 & 4.3 & 2 & 0.89 & 0.82 & -0.24 & 5.57 & 15.8 & 2.5 \\
\hline & 80 & 0.10 & 0.07 & 0.05 & 0.20 & & 1.0 & & 0.05 & 0.03 & & 0.06 & 2.2 & \\
\hline \multirow[t]{2}{*}{ HD 112196} & 6110 & $4.39^{1}$ & 0.01 & 0.02 & 1.31 & 4.90 & 12.0 & 1 & 1.15 & 1.14 & -0.09 & 4.22 & - & - \\
\hline & 60 & 0.06 & 0.05 & 0.05 & 0.20 & & 1.0 & & 0.05 & 0.08 & & 0.14 & - & \\
\hline \multirow[t]{2}{*}{ HD 167389} & 5895 & 4.37 & -0.02 & -0.03 & 0.99 & 4.00 & 3.3 & 1 & 1.05 & 1.01 & -0.12 & 4.64 & 36.8 & 10.0 \\
\hline & 80 & 0.15 & 0.07 & 0.05 & 0.20 & & 1.0 & & 0.05 & 0.04 & & 0.07 & 6.5 & \\
\hline \multirow[t]{2}{*}{ HD 184960} & 6310 & 4.22 & -0.08 & -0.01 & 1.55 & 5.70 & 8.0 & 1 & 1.23 & 1.43 & -0.08 & 3.59 & 25.6 & 0.0 \\
\hline & 80 & 0.10 & 0.05 & 0.05 & 0.20 & & 1.0 & & 0.05 & 0.05 & & 0.06 & 3.1 & \\
\hline \multicolumn{15}{|c|}{$M 01 k, K 03 k, K 03 f$} \\
\hline \multirow[t]{2}{*}{ HD 59747} & 5094 & 4.55 & -0.03 & -0.01 & 1.00 & 0.90 & 1.5 & 2 & 0.83 & 0.76 & -0.31 & 5.90 & 20.8 & 5.8 \\
\hline & 90 & 0.10 & 0.07 & 0.05 & 0.20 & & 1.0 & & 0.05 & 0.04 & & 0.08 & 2.9 & \\
\hline \multirow[t]{2}{*}{ HD 109647} & 4980 & 4.70 & 0.05 & -0.07 & 0.80 & 0.40 & 3.1 & 1 & 0.83 & 0.74 & -0.36 & 6.05 & 24.0 & -8.8 \\
\hline & 60 & 0.15 & 0.15 & 0.15 & 0.20 & & 1.0 & & 0.05 & 0.03 & & 0.08 & 4.3 & \\
\hline \multirow[t]{2}{*}{ HD 110463} & 4940 & 4.68 & 0.00 & -0.02 & 0.86 & 0.40 & 3.1 & 1 & 0.80 & 0.75 & -0.38 & 6.05 & 20.9 & -9.8 \\
\hline & 80 & 0.18 & 0.10 & 0.10 & 0.20 & & 1.0 & & 0.05 & 0.03 & & 0.07 & 4.5 & \\
\hline \multirow[t]{2}{*}{ HD 115043} & 5850 & 4.36 & -0.10 & 0.05 & 1.26 & 3.90 & 7.5 & 1 & 1.05 & 1.03 & -0.13 & 4.64 & 28.3 & 9.3 \\
\hline & 70 & 0.13 & 0.07 & 0.05 & 0.20 & & 1.0 & & 0.05 & 0.04 & & 0.06 & 4.4 & \\
\hline \multicolumn{15}{|l|}{$K 03 k, K 03 f$} \\
\hline \multirow[t]{2}{*}{ HD 28495} & 5465 & 4.45 & -0.05 & 0.00 & 0.93 & 2.34 & 4.5 & 1 & 0.95 & 0.85 & -0.19 & 5.35 & 31.3 & 13.8 \\
\hline & 90 & 0.12 & 0.05 & 0.05 & 0.20 & & 1.0 & & 0.10 & 0.04 & & 0.08 & 4.8 & \\
\hline \multirow[t]{2}{*}{ HD 39587} & 5920 & 4.39 & -0.07 & -0.01 & 0.95 & 4.20 & 8.7 & 2 & 1.04 & 1.03 & -0.12 & 4.59 & 9.1 & 5.3 \\
\hline & 70 & 0.10 & 0.07 & 0.05 & 0.20 & & 0.8 & & 0.05 & 0.04 & & 0.05 & 1.3 & \\
\hline \multirow{2}{*}{ HD $71974 A^{2}$} & 5570 & 4.49 & - & - & - & 2.8 & 3.2 & 1 & - & - & - & - & - & - \\
\hline & 100 & 0.19 & - & - & - & & 1.0 & & - & - & & - & - & \\
\hline \multirow[t]{2}{*}{ HD 109011} & 5000 & 4.60 & -0.12 & -0.03 & $\lessgtr 0.52$ & 0.50 & 5.0 & 1 & 0.83 & 0.81 & -0.34 & 5.85 & 22.3 & -6.0 \\
\hline & 100 & 0.20 & 0.07 & 0.07 & - & & 1.0 & & 0.05 & 0.07 & & 0.17 & 5.3 & \\
\hline \multirow[t]{2}{*}{ HD 171746 A } & 6100 & $4.36^{1}$ & -0.04 & 0.03 & 1.50 & 4.80 & 8.3 & 1 & 1.15 & 1.18 & -0.10 & 4.16 & - & - \\
\hline & 80 & 0.06 & 0.06 & 0.05 & 0.20 & & 1.0 & & 0.05 & 0.08 & & 0.14 & - & \\
\hline \multirow[t]{2}{*}{ HD 171746 B } & 6040 & $4.37^{1}$ & 0.02 & -0.04 & 1.51 & 4.60 & 7.3 & 1 & 1.10 & 1.16 & -0.10 & 4.24 & - & - \\
\hline & 80 & 0.06 & 0.05 & 0.05 & 0.20 & & 1.0 & & 0.05 & 0.08 & & 0.14 & - & \\
\hline
\end{tabular}

The stellar parameters of the sample stars which were studied following the methods of Fuhrmann (1998). The stars are grouped according to the samples they form part of (Table 3). The following quantities are listed with the second line providing the errors bars: (2) effective temperature, (3) surface gravity, (4) iron abundance, (5) magnesium abundance relative to iron, (6) microturbulence parameter, (7) macroturbulence parameter, (8) projected rotational velocity, (9) source of the values: 1 = this work, 2 = F04, $3=$ König et al. (2006), (10) mass, (11) radius, (12) bolometric correction based on Alonso et al. (1995), (13) absolute bolometric magnitude (from Hipparcos distance), (14) spectroscopic distance, and (15) relative offset from Hipparcos distance.

${ }^{1}$ Surface gravity derived from Hipparcos parallax as described in Sect. 3.1.4. The comparison of spectroscopic distance with Hipparcos distance cannot be done.

2 The stellar parameters are preliminary because HD 71974 is a spectroscopically unresolved binary (see discussion of individual objects in Sect. 3.3). Abundances could not be derived.

gravity is calculated from Hipparcos parallaxes $\pi$ (for details see Fuhrmann et al. 1997b; Ammler 2006a,b):

$$
\begin{aligned}
\log g= & 2 \log \pi+4.44+\log M+4 \log \frac{T_{\mathrm{eff}}}{5780 \mathrm{~K}} \\
& +\frac{2}{5}\left(V-A_{\mathrm{V}}-B . C \cdot \mathrm{V}+5-M_{\mathrm{bol}, \odot}\right),
\end{aligned}
$$

where $\pi$ is the parallax. The apparent visual magnitude $V$ is derived from the Hipparcos $H_{\mathrm{P}}$ magnitudes using the conversion tables of Bessell (2000). The adopted parallaxes and magnitudes are given in Table 3. The bolometric corrections B.C.v are taken from Alonso et al. (1995) $)^{9}$. All of the stars analysed in this work are closer than $40 \mathrm{pc}$ so that interstellar absorption is considered

9 They use another definition of B.C.v, which has an opposite sign! neglible and not accounted for $\left(A_{\mathrm{V}}=0\right)$. The stellar mass $M$ finally is given in units of solar mass. Since the UMa group has an age of $\approx 10^{8}$ Myr and all sample stars have spectral types from late-FV to early-KV, mass is estimated by simply comparing the stellar parameters to the theoretical zero-age main sequence of VandenBerg et al. $(2000)([\mathrm{Fe} / \mathrm{H}]=0.00,[\alpha / \mathrm{Fe}]=0.0)$.

An error bar is obtained from Gaussian error propagation using partial derivatives (see Tables 3 and 5 for the individual contributions entering Eq. (1)). While photometric variability would lead to larger errors, we expect that it is low (cf. Adelman et al. 2000) for the warmer stars addressed here. The resulting gravity error bars are 0.06 dex or smaller.

Finally, iron abundance and microturbulence are derived from Fe II lines. Errors are inferred again by varying effective temperature within its error bar. 
Table 6. Li absorption and $\mathrm{H} \alpha$ infilling.

\begin{tabular}{|c|c|c|c|c|c|c|c|c|c|c|}
\hline Object & $\begin{array}{l}T_{\text {eff }} \\
{[\mathrm{K}]}\end{array}$ & $\begin{array}{c}W_{\mathrm{Li}}^{\text {int }} \\
{[\mathrm{m \AA}]}\end{array}$ & $\begin{array}{c}W_{\mathrm{Li}}^{\mathrm{fit}} \\
{[\mathrm{m \AA}]}\end{array}$ & $\begin{array}{c}W_{\mathrm{Li}}^{\mathrm{KS} 05} \\
{[\mathrm{~mA}]}\end{array}$ & $\log N_{\text {LTE }}^{\operatorname{cog}}(\mathrm{Li})$ & $\log N_{\text {NLTE }}^{\operatorname{cog}}(\mathrm{Li})$ & $\log N_{\mathrm{LTE}}^{\mathrm{fit}}(\mathrm{Li})$ & $\log N_{\mathrm{LTE}}^{\mathrm{KS} 05}(\mathrm{Li})$ & $\begin{array}{c}\text { H } \alpha \text { core } \\
{[\%]}\end{array}$ & $F_{\mathrm{H} \alpha}\left[10^{5} \mathrm{erg} \mathrm{cm}^{-2} \mathrm{~s}^{-1}\right]$ \\
\hline \multicolumn{11}{|l|}{ M01k } \\
\hline HD 11131 & $5796^{c}$ & $70.5 \pm 1.9$ & 71.2 & 78.0 & $2.47 \pm 0.06$ & $2.46 \pm 0.06$ & $2.58 \pm 0.10$ & $2.38 \pm 0.04$ & $29^{c}$ & 10.87 \\
\hline HD $24916 \mathrm{~A}$ & $4600^{b}$ & $<8.0 \pm 3.0$ & & & & & & & 33 & 1.08 \\
\hline HD 26923 & $5975^{c}$ & $88.0 \pm 2.1$ & 88.9 & 84.3 & $2.77 \pm 0.07$ & $2.72 \pm 0.06$ & $2.83 \pm 0.09$ & $2.69 \pm 0.03$ & $25^{c}$ & 9.02 \\
\hline HD 38393 & 6310 & $65.3 \pm 0.7$ & 63.5 & 58.5 & $2.87 \pm 0.05$ & $2.81 \pm 0.04$ & $2.96 \pm 0.09$ & $2.82 \pm 0.03$ & 17 & 0.33 \\
\hline HD 41593 & $5278^{c}$ & $14.0 \pm 3.9$ & 15.8 & & $1.17 \pm 0.16$ & $1.29 \pm 0.15$ & $1.20 \pm 0.12$ & & $30^{c}$ & 8.72 \\
\hline HD 238087 & $4350^{g}$ & $<20.0 \pm 5.0$ & & & & & & & 39 & - \\
\hline HD 112196 & 6110 & $109.8 \pm 3.1$ & 109.5 & & $3.04 \pm 0.06$ & $2.94 \pm 0.05$ & $3.09 \pm 0.09$ & & 28 & 11.17 \\
\hline HD $152863 \mathrm{~A}^{a}$ & $4980^{e}$ & $<7.0 \pm 5.0$ & & & & & & & 17 & -0.37 \\
\hline HD $155674 \mathrm{~A}$ & $\lesssim 4800$ & $<9.0 \pm 6.0$ & & & & & & & 37 & 11.87 \\
\hline HD $155674 \mathrm{~B}$ & $3900^{f}$ & $<10.0 \pm 2.0$ & & & & & & & 39 & - \\
\hline HD 167389 & 5895 & $59.7 \pm 2.4$ & 59.2 & 58.8 & $2.48 \pm 0.08$ & $2.46 \pm 0.07$ & $2.50 \pm 0.10$ & $2.41 \pm 0.04$ & 21 & 4.70 \\
\hline HD 184960 & 6310 & $60.5 \pm 1.9$ & 60.0 & 61.6 & $2.82 \pm 0.04$ & $2.76 \pm 0.04$ & $2.90 \pm 0.08$ & $2.78 \pm 0.03$ & 17 & -0.02 \\
\hline HD $205435^{a}$ & $5060^{g}$ & $<15.0 \pm 5.0$ & & & & & & & 20 & -0.73 \\
\hline \multicolumn{11}{|l|}{$M 01 k, K 03 k$} \\
\hline HD 238224 & $4350^{f}$ & $<11.0 \pm 4.0$ & & & & & & & 61 & - \\
\hline \multicolumn{11}{|c|}{$M 01 k, K 03 k, K 03 f$} \\
\hline HD 59747 & $5094^{c}$ & $35.8 \pm 4.4$ & 38 & 41.0 & $1.39 \pm 0.12$ & $1.53 \pm 0.10$ & $1.44 \pm 0.16$ & $1.16 \pm 0.07$ & $32^{c}$ & 9.90 \\
\hline HD 109647 & 4980 & $46.0 \pm 5.0$ & 44.2 & 25.0 & $1.37 \pm 0.09$ & $1.53 \pm 0.08$ & $1.36 \pm 0.11$ & $0.49 \pm 0.15$ & 34 & 11.65 \\
\hline HD 110463 & 4940 & $21.8 \pm 2.6$ & 23.7 & 18.0 & $0.99 \pm 0.11$ & $1.17 \pm 0.10$ & $1.05 \pm 0.11$ & $0.31 \pm 0.06$ & 32 & 10.03 \\
\hline HD 115043 & 5850 & $94.0 \pm 6.0$ & 84.0 & 101 & $2.70 \pm 0.08$ & $2.66 \pm 0.06$ & $2.78 \pm 0.11$ & $2.61 \pm 0.08$ & 27 & 9.48 \\
\hline \multicolumn{11}{|l|}{$K 03 k, K 03 f$} \\
\hline HD 28495 & 5465 & $69.0 \pm 2.4$ & 72.8 & 71.5 & $2.14 \pm 0.09$ & $2.18 \pm 0.07$ & $2.21 \pm 0.08$ & $1.86 \pm 0.05$ & 42 & 20.43 \\
\hline HD 39587 & $5920^{c}$ & $103.6 \pm 4.4$ & 104.7 & 102.8 & $2.83 \pm 0.08$ & $2.77 \pm 0.07$ & $2.91 \pm 0.10$ & $2.74 \pm 0.02$ & $28^{c}$ & 11.14 \\
\hline HD 71974 A & 5570 & $68.0 \pm 4.0$ & 66.7 & & & & & & 32 & 12.90 \\
\hline HD 95650 & $3634^{d}$ & $<45.0 \pm 5.0$ & & & & & & & 73 & - \\
\hline HD 109011 & 5000 & $29.0 \pm 6.0$ & 25.9 & 23.4 & $1.19 \pm 0.16$ & $1.35 \pm 0.14$ & $1.22 \pm 0.20$ & $0.55 \pm 0.04$ & 36 & 12.54 \\
\hline HD $171746 \mathrm{~A}$ & 6100 & $84.9 \pm 3.5$ & 83.3 & & $2.85 \pm 0.08$ & $2.79 \pm 0.07$ & $2.90 \pm 0.10$ & & 17 & 10.34 \\
\hline HD $171746 \mathrm{~B}$ & 6040 & $50.0 \pm 5.0$ & 45.3 & & $2.51 \pm 0.09$ & $2.48 \pm 0.08$ & $2.51 \pm 0.11$ & & 27 & 7.26 \\
\hline
\end{tabular}

(2) Effective temperature is adopted from Table 5 if available, or from other sources as indicated in the footnotes to the table. (3) Equivalent width $W_{\mathrm{Li}}^{\text {int }}$ of Li I $\lambda 6707.8 \AA$ from direct integration of the observed profile. (4) Equivalent width $W_{\mathrm{Li}}^{\text {fit }}$ from fitting with synthetic line profiles. (5) Equivalent width $W_{\mathrm{Li}}^{\mathrm{KS} 05}$ according to King \& Schuler (2005). (6) $\mathrm{Li}$ abundance $\log N_{\mathrm{LTE}}^{\mathrm{cog}}(\mathrm{Li})$ using $W_{\mathrm{Li}}^{\text {int }}$ by interpolation of the curves of growth of Soderblom et al. (1993a). (7) After application of the non-LTE corrections of Carlsson et al. (1994) (log $N_{\mathrm{NLTE}}^{\operatorname{cog}}(\mathrm{Li})$ ). (8) Li abundance $\log N_{\mathrm{LTE}}^{\mathrm{fit}}(\mathrm{Li})$ derived by fitting a synthetic profile to the observed feature. (9) Li abundance $\log N_{\mathrm{LTE}}^{\mathrm{KS} 05}(\mathrm{Li})$ according to King \& Schuler (2005). (10) Relative infilling of the core of $\mathrm{H} \alpha$ measured according to F04. (11) Chromospheric flux $F_{\mathrm{H} \alpha}$ measured according to Lyra \& Porto de Mello (2005).

${ }^{a}$ Giant. ${ }^{b}$ From König et al. (2006). ${ }^{c}$ From F04. ${ }^{d}$ From Alonso et al. (1996). ${ }^{e}$ From de Laverny et al. (2003). ${ }^{f}$ Converted from spectral type with the calibration from Kenyon \& Hartmann (1995). ${ }^{g}$ From McWilliam (1990).

\subsubsection{Instrumental profile, macroturbulence, and rotational velocity}

The macroturbulence parameter is calculated from the linear fit given by Gray (1984)

$\zeta_{\mathrm{RT}}\left[\mathrm{km} \mathrm{s}^{-1}\right]=3.95 \cdot T_{\mathrm{eff}}\left[10^{3} \mathrm{~K}\right]-19.25$.

The instrumental profile is obtained by convolution with the Kitt Peak solar-flux atlas (Kurucz et al. 1984) and comparison to the observed lunar spectrum. The projected rotational velocity $v \sin i$ is then inferred by fitting individual weak Fe II lines. The scatter of $v \sin i$ is usually less than $0.50 \mathrm{~km} \mathrm{~s}^{-1}$. However, taking into account the uncertainties in both the instrumental broadening and the macroturbulence parameter, the true error is probably closer to $1.0 \mathrm{~km} \mathrm{~s}^{-1}$.

\subsection{Accuracy of the stellar parameters}

All results are listed in Table 5. Although error bars are estimated for all parameters, accounting for all possible systematic and random errors is hardly feasible. Therefore, accuracy is judged by several tests. The first test is a consistency test. Since the method works differentially with respect to the Sun, the analysis of the observed solar spectra has to - and does - reproduce the parameters of the Sun (see Ammler 2006a,b, for details). The second test is simply to analyse a star that has already been studied previously by F04, which in our work is HD 217813. This star is well suited to this comparison since it is a probable UMa group member. The values that we derived are consistent with those previously found (see Ammler et al. 2005).

A crucial test of the spectroscopically derived surface gravities is the comparison of the spectroscopic distance with the Hipparcos distance (Fig. 2). Spectroscopic distance is calculated by solving Eq. (1) for the parallax using the stellar parameters derived (Table 5), and the remaining quantities as described in Sect. 3.1.4. Of course HD 38393, HD 112196, HD 171746 A, and HD 171746 B are not included in this comparison, because their surface gravities were calculated from the Hipparcos distances.

The error bars are calculated by Gaussian error propagation using the partial derivatives of Eq. (1). At first glance, the spectroscopic distances agree very well with the Hipparcos distances within the error bars, but a closer look identifies subtle differences, which are interesting to discuss. First of all, the scatter of the new measurements is noticeably larger than that in F04 and König et al. (2006). However, this is not surprising since the methods are applied here to stars that are cooler, as well as hotter, than those in these previous studies. The presently studied 


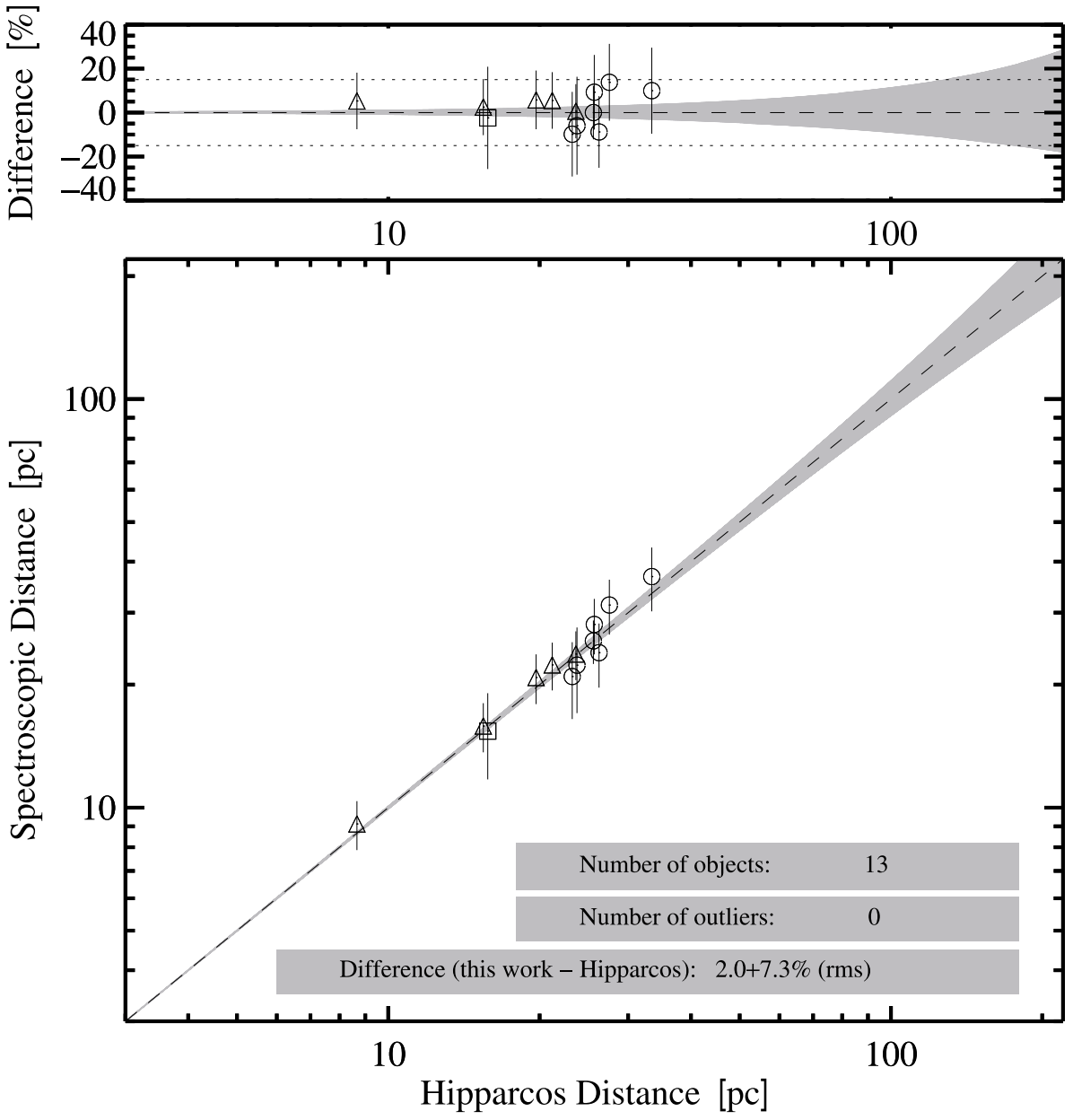

Fig. 2. Comparison of spectroscopic distances with Hipparcos distances - The bottom panel allows one to directly compare the distances. The dashed line represents perfect agreement with Hipparcos. The grey-shaded area displays the Hipparcos errors. The top panel shows the residuals and allows one to judge the overall accuracy of the spectroscopic distances. The dotted line represents the typical individual uncertainties of $10-15 \%$ experienced by F04. Data from our work are depicted by circles, whereas the data from F04 are denoted by triangles. The square represents the spectroscopic distance of HD 24916 A, which was calculated from the parameters given by König et al. (2006). stars are at the edge of the temperature regime where the applied methods can be used. At very cool temperatures, the number of Fe II-lines becomes small ${ }^{10}$ and MAFAGS models of cool metalrich atmospheres are unavailable. In the case of the hot stars, the determination of the surface gravities from the ionization equilibrium of the Fe I and Fe II lines is complicated. It is thus unsurprising that stars with very low, or very high temperatures are less well modelled. Another point that should be kept in mind is binarity. A binary, in which both stars have the same brightness, would have a greater spectroscopic distance by up to $41 \%$. Since the luminosity of the object is needed to derive the spectroscopic distance, the distance of a binary will always be found to be too large. A close inspection of Fig. 2 indicates that there are three stars for which the spectroscopic distance is a bit too large, and these objects are discussed in more detail in the next section. Apart from these subtle differences, the agreement between the spectroscopic distance and the Hipparcos distance is fairly good so that the stellar parameters derived in the present work are considered to be correct.

\subsection{The binary nature of individual objects}

The confusion between single and binary stars may produce spurious stellar parameters. In the following, the possible influences of multiplicity on the spectroscopic analyses of HD 28495,

\footnotetext{
${ }_{10}$ The few available Fe II lines were checked for blends using the $e x$ tract stellar feature of VALD (Vienna Astrophysical Line Data-Base) (Kupka et al. 2000, 1999; Ryabchikova et al. 1997; Piskunov et al. 1995 ) with a sensitivity limit of 0.05 .
}

HD 115043, and HD 167389 are discussed, stars which all have a somewhat too large spectroscopic distance in Fig. 2, as well as the visual binary HD 171746 and the unresolved binary HD 71974 A.

- HD 28495: Until now, this star has always been referred to as a single star. However, the catalogue of Makarov \& Kaplan (2005) lists a difference of $18.3 \pm 2.3$ mas/yr in the proper motion measurements of Hipparcos and Tycho-2, and an acceleration of the proper motion of $17.8 \pm 2.8 \mathrm{mas} / \mathrm{yr}^{2}$. Following the recipe given in Makarov \& Kaplan (2005) and assuming a circular orbit, an upper limit to the mass of a hypothetical companion of $0.20 M_{\odot}$ was derived. This object would have an absolute $V$-band magnitude $M_{V}=12$, and would thus be 6.5 mag fainter than the primary (Baraffe et al. 1998, Fig. 3). This faint companion would certainly not spoil the analysis presented in the present work. It is thus more plausible that the analysis - in particular the temperature determination - was slightly affected by the partly infilled $\mathrm{H} \alpha$ profile.

- HD71974 A: The resulting effective temperature and surface gravity may be spurious, since the object is a close binary that cannot be resolved with spectroscopy. Fabricius \& Makarov (2000) give a separation of 0 ". 48 and a brightness difference of $\Delta H_{\mathrm{P}}=1.6$, while Heintz (1988) find a separation of 0 ". 377 and a brightness difference of $1.4^{11}$.

- HD 115043: Lowrance et al. (2005) detected a companion of spectral type M4-M5 at a separation of 1".58. Given this

11 Photometric band not given. 

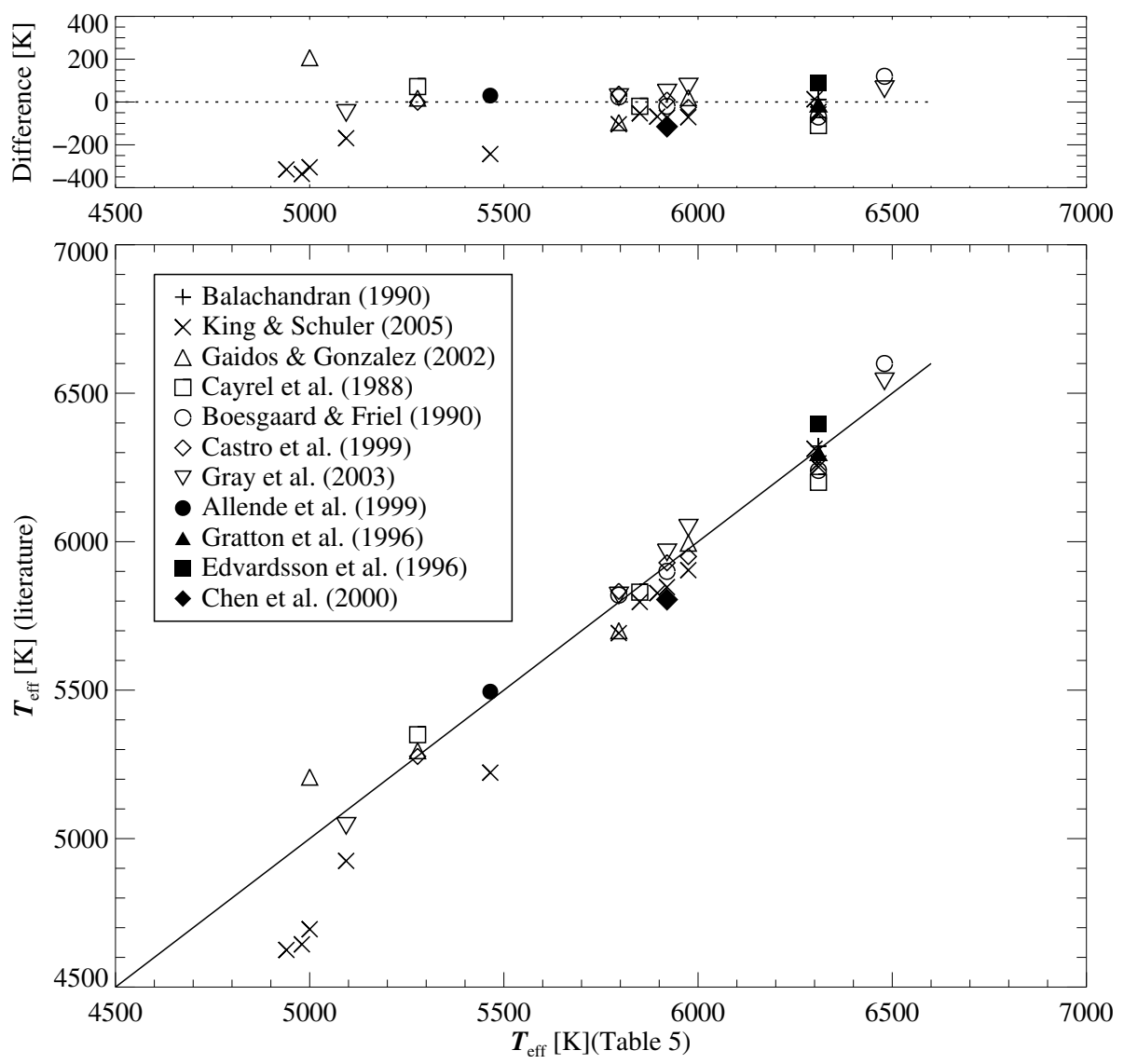

Fig. 3. Comparison with effective temperatures from previous analyses - the solid line indicates equality of the literature values and the effective temperatures presented in Table 5. HD 111456 and HD 134083 are added for completeness. They are fast-rotating UMa group members with effective temperature estimates given by Fuhrmann (2000) $\left(T_{\text {eff }}=6300 \mathrm{~K}\right.$ and $T_{\text {eff }}=6480 \mathrm{~K}$, resp.). Error bars are omitted for clarity.

spectral type, the absolute $V$ band magnitude of the companion is $M_{V} \sim 11.8$ (Schmidt-Kaler 1982, Table 3), compared to $M_{V}=4.63$ of the primary. Again, ths faint companion would not have affected the spectroscopic analysis.

- HD 167389: According to all sources listed in SIMBAD, this object is a single star. However, the fits to the $\mathrm{Mg}$ Ib lines are insufficient possibly leading to an underestimation of the surface gravity.

- HD 171746 A \& B: The two components of this binary have a separation of 1 '.718. The position angle is 158.8 (Fabricius \& Makarov 2000). Using the photometry from Fabricius \& Makarov (2000) and converting the Hipparcos system to the Johnson V-system (Bessell 2000), the A and $\mathrm{B}$ components have a brightness of $V_{\mathrm{A}}=6.924 \pm 0.071$ and $V_{\mathrm{B}}=7.009 \pm 0.071$. Because of the small separation and the nearly equal brightness of the two stars, some light from the B component may have entered the fibre, when the A component was observed, and of course light from the A component may have entered when the $\mathrm{B}$ component was observed. Thus, the resulting stellar parameters should be regarded with care.

\subsection{Comparison of stellar parameters with previous studies}

Since a thorough comparison of our work with results obtained by other authors has already been presented in Ammler (2006a,b), only the most meaningful findings are discussed here. The comparison with King \& Schuler (2005) is of particular interest, because they have several stars in common with the present work. Furthermore, King \& Schuler (2005) used LTE model atmospheres, and their method is also differential with respect to the Sun. However, their derivation of stellar parameters differed from our work. They determined effective temperatures from a colour-based calibration (Steinhauer 2003) and used surface gravities derived from evolutionary models of Yi et al. (2001). Finally, King \& Schuler (2005) adopted the microturbulence parameter from the relation given in Allende Prieto et al. (2004).

Figure 3 shows a comparison of the effective temperatures given in Table 5 with those derived in previous studies. All values - except those of King \& Schuler (2005) - scatter closely around the solid line, which indicates equality. This and the good agreement of spectroscopic distance with the geometric Hipparcos distance generally provides confidence in the temperatures derived in the present work. A systematic discrepancy with the values derived by King \& Schuler (2005) can be noted in Fig. 3. This difference increases towards the lowest effective temperatures and is probably caused by the adoption of an external calibration by King \& Schuler (2005).

The abundances derived depend strongly on the value of the microturbulence parameter used within the synthetic line formation. The iron abundance is underestimated if too large a value of the microturbulence parameter is adopted. Similar to previous studies (see Fuhrmann 2004; Luck \& Heiter 2005), an increase in the microturbulence parameter with increasing effective temperatures is found (Fig. 4). In the present case, the increase is yet higher because of the hotter stars in the sample. Since the exact value of the microturbulence parameter is determined by the way in which the analysis was done, the only meaningful comparison is that with the work of F04, as illustrated by the different values quoted for the Sun. Fuhrmann (1998) gives $0.90 \pm 0.15 \mathrm{~km} \mathrm{~s}^{-1}$, whereas Allende Prieto et al. (2004) adopts $1.25 \mathrm{~km} \mathrm{~s}^{-1}$. In the present work, solar spectra of different observing runs are analysed and an average of $0.88 \pm 0.06 \mathrm{~km} \mathrm{~s}^{-1}$ is obtained, which has to and indeed does agree excellently with F04. The lower panel of Fig. 4 displays the ratio $\frac{\zeta_{\mathrm{RT}}}{\xi_{\mathrm{t}}}$ versus effective temperature, 

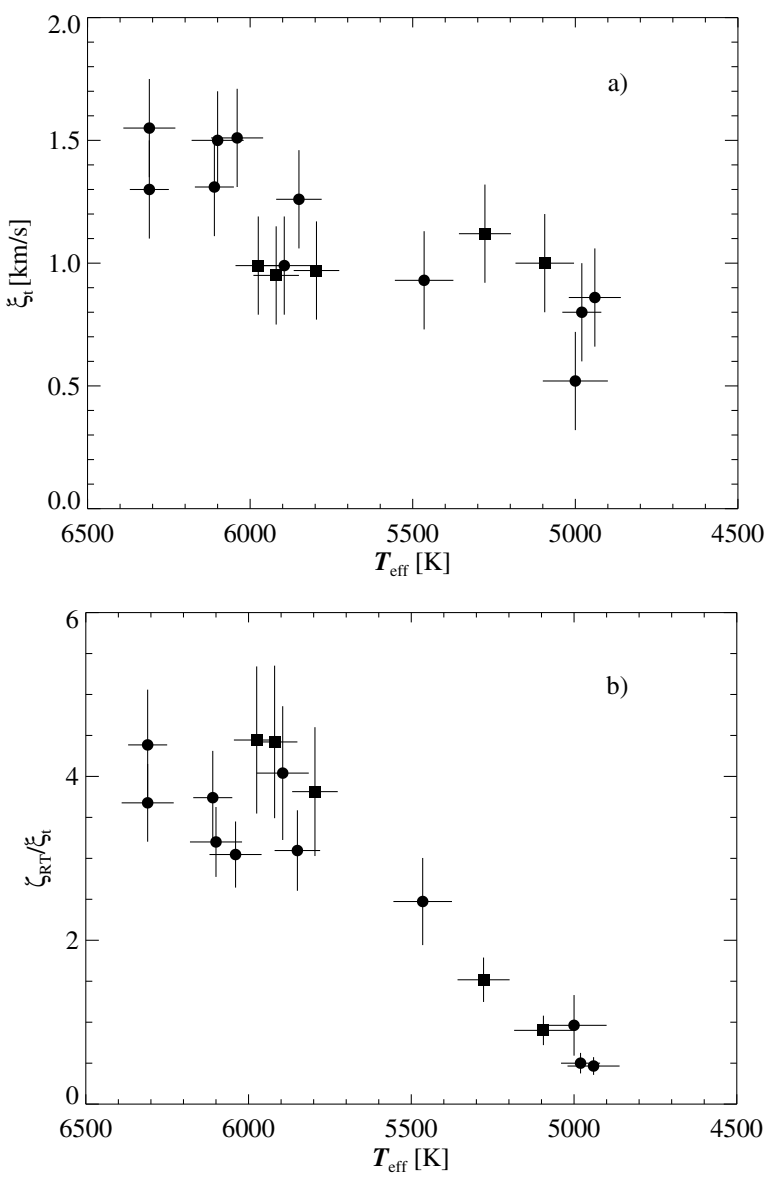

Fig. 4. Comparison of the microturbulence parameter $\xi_{\mathrm{t}}$ with F04 - all values are taken from Table 5. a) The values of the microturbulence parameter derived in the present work (circles) generally follow the trend with effective temperature of the values determined by F04 (squares). b) $\zeta_{\mathrm{RT}} / \xi_{\mathrm{t}}$ is displayed vs. efffective temperature. Symbols are the same as in the upper panel.

which can be compared to Fuhrmann (2004, Fig. 5). The systematic difference at hotter temperatures cannot be fully explained. While somewhat higher values of the microturbulence parameter are expected in this temperature regime (Fuhrmann 2004; Gaidos \& Gonzalez 2002), the values found in the present work (circles) follow the trend of old stars in the lower panel of Fig. 4 whereas the data adopted from F04 (squares) trace the trend of the young stars according to their Fig. 5.

\section{Spectroscopic properties of the Ursa Major group}

\subsection{Distribution of iron and magnesium abundance}

Table 5 implies an average iron abundance of $\langle[\mathrm{Fe} / \mathrm{H}]\rangle=$ $-0.03 \pm 0.05$ dex, for the kinematic members identified by M01 (sample $M 01 K)$. The value for both the kinematic $(K 03 k)$ and final members $(K 03 f)$ of $\mathrm{K} 03$ is $\langle[\mathrm{Fe} / \mathrm{H}]\rangle=-0.04 \pm 0.05$ dex because of both the kinematic and final members of K03 in Table 5 are identical. Therefore, the mean abundances of all three samples $M 01 k, K 03 k$, and $K 03 f$ are almost the same making the definition of a mean UMa group iron abundance very robust.

The values are slightly higher than previous measurements $(-0.08 \pm 0.09$, Soderblom \& Mayor 1993b; $-0.09 \pm 0.02$, Boesgaard \& Friel 1990). The abundance measurements of King \& Schuler (2005) for stars in common imply a mean of $-0.06 \pm 0.08$. King \& Schuler (2005) already highlighted the small abundance scatter of the UMa group. The spread measured in the present work is even smaller than the scatter found by King \& Schuler (2005). Even more, it is within the error bars of individual measurements, i.e., the iron abundances of the UMa group members are almost indistinguishable.

It is interesting to compare the iron abundances derived for the UMa group with other young stellar clusters. The Hyades are metal-rich according to Boesgaard \& Budge $(1988)([\mathrm{Fe} / \mathrm{H}]=$ $+0.17 \pm 0.06)$, Cayrel et al. $(1985)([\mathrm{Fe} / \mathrm{H}]=+0.12 \pm 0.03)$, Paulson et al. $(2003)([\mathrm{Fe} / \mathrm{H}]=+0.13 \pm 0.01)$, and Boesgaard \& Friel (1990) $([\mathrm{Fe} / \mathrm{H}]=+0.13 \pm 0.05)$. Boesgaard \& Friel $(1990)$ derive a value of $[\mathrm{Fe} / \mathrm{H}]=-0.03 \pm 0.02$ for the Pleiades. After removing known non-members from their sample (An et al. 2007), the metallicity is found to be higher, $[\mathrm{Fe} / \mathrm{H}]=+0.03$. This slightly super-solar value is also supported by the results of King et al. (2000) $([\mathrm{Fe} / \mathrm{H}]=+0.06 \pm 0.05)$ and Grupp (2004) $([\mathrm{Fe} / \mathrm{H}]=+0.04 \pm 0.02)$. All in all, the iron abundance of the UMa group is about the same as that of the Pleiades, and both have about the solar abundance. Taking into account magnesium (Table 5), the distribution of chemical abundances coincides well with the thin disk population (according to Fuhrmann 2004, Fig. 34). The scatter of the UMa group Fe abundance is also at most $10 \%$ of the total thin disk Fe abundance spread.

In an exclusive sense, a membership criterion can be obtained from the measured mean, defining the range of allowed values by $2 \sigma$. Thus, an $\mathrm{Fe}$ abundance of $[\mathrm{Fe} / \mathrm{H}]=-0.04 \pm 0.10$ is expected for any UMa group member.

\subsection{Youth and activity}

\subsubsection{Lithium absorption}

Table 6 tabulates lithium abundances and equivalent widths measured for the LiI $\lambda 6708 \AA$ feature. The observed spectroscopic data is supplemented by spectra kindly provided by K. Fuhrmann for HD 11131, HD 26923, HD 39587, HD 41593 , and HD 59747.

The measurements follow two different approaches. Firstly, the lithium equivalent widths are assessed by integrating the observed profiles. Using these data, abundance is derived in the same way as Sestito \& Randich (2005), i.e., by interpolation of the LTE curves of growth of Soderblom et al. (1993a, Table 2) and by applying the non-LTE (NLTE) corrections given by Carlsson et al. (1994) ${ }^{12}$. Secondly, the lithium abundances are determined by fitting the observed lithium feature with a synthetic profile to check the consistency with the curve-of-growth method. The synthetic Li profile is calculated in the same way as the $\mathrm{Fe}$ and $\mathrm{Mg}$ lines described before using the line data in Table 4. Equivalent widths are not needed within this second approach but the equivalent width of the matching synthetic profile is given to compare to the observed values. Lithium abundance is only provided for the stars with uniformly derived stellar parameters (Table 5), so that additional systematics are not introduced and the relative accuracy of the results among the sample is preserved.

The quality of the measured equivalent widths strongly depends on the resolution and the signal-to-noise ratio of the underlying spectra. In the present work, a signal-to-noise ratio of at least $\approx 200$ with a resolving power of $\approx 60000$ was achievable for almost all stars. The blend with the Fe I line is mostly resolved.

\footnotetext{
12 Instead of interpolating their Table 1, the data rows for metal-rich dwarfs are adopted, that is for a surface gravity of $\log g=4.5$ and an iron abundance of $[\mathrm{Fe} / \mathrm{H}]=0$.
} 

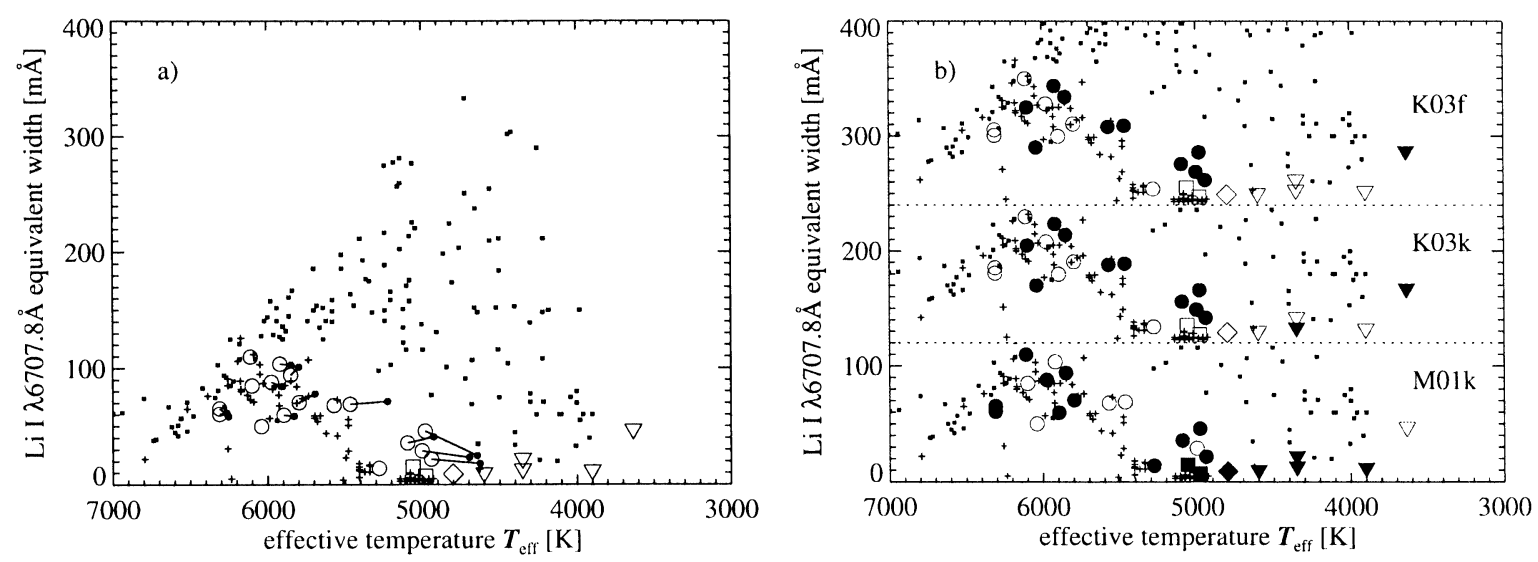

Fig. 5. Effective temperature vs. lithium absorption - a) The uniform measurements of this work (open symbols, Table 6 , $W_{\mathrm{Li}}^{\text {int }}$ ) are compared to previous determinations by King \& Schuler (2005) for all stars in common (dots, connected by lines, Table 6, $W_{\mathrm{Li}}^{\mathrm{KS} 05}$ ). Furthermore, the distribution of the Pleiades (filled squares) and Hyades (crosses) is shown by adopting the data from Sestito \& Randich (2005). The measurements of this work are depicted by circles, while upper limits are given by triangles and in the cases of the two giants HD 152863 A and HD 205435 by squares. In the case of HD $155674 \mathrm{~A}$, the diamond depicts upper limits both to Li equivalent width and effective temperature. Error bars are omitted for clarity. b) The distributions are repeated for the different samples $M 01 k, K 03 k$, and $K 03 f$ according to Table 3, using arbitrary offsets. The dotted lines indicate an equivalent width of zero to aid the eye. The members of each sample are depicted by filled symbols.

Therefore, the present equivalent widths of the UMa group can be considered to be very accurate. Uncertainties in the equivalent widths from direct integration are found by varying the integration limits and the position of the continuum, which depends on both noise and blends with neighbouring lines. Uncertainties in the curve-of-growth abundances are assessed by varying the input parameters, i.e., the equivalent widths, effective temperatures, and surface gravities within their error bars. The resulting total formal error amounts to typically less than $\approx 0.10$ dex.

Similarly, the synthetic line fits are repeated by varying the stellar parameters within their error bars. The variation in both effective temperature and iron abundance dominated the changes in lithium abundance, so that only variations in these two parameters are considered. The final error again generally equals to $\approx 0.10$ dex.

The equivalent widths emerging from these methods agree remarkably well, except in the case of HD 115043. The abundances from the LTE curves-of-growth should be consistent with the values obtained by the LTE line synthesis. Considering the problem of line blends, Hünsch et al. (2004) also derived $\mathrm{Li}$ abundance with both methods of synthetic line fits and curves of growth. Following their Fig. 3, the values from synthetic fits exceeded those from curves of growth (Soderblom et al. 1993a) by $0.1-0.3$ dex. This effect can be reproduced from Table 6 , although it is lower in magnitude. In the present work, the average difference $\left\langle\log N_{\text {LTE }}^{\mathrm{cog}}(\mathrm{Li})-\log N_{\mathrm{LTE}}^{\mathrm{fit}}(\mathrm{Li})\right\rangle=-0.05 \pm 0.03$ can be fully explained by the slightly sub-solar mean iron abundance of the UMa group and the neglected influence of iron abundance in the curve of growth tables of Soderblom et al. (1993a). Completing line synthesis with increased iron abundance shows that $\mathrm{Li}$ abundance needs to be reduced by approximately the same amount to fit the observed profile. Yet, a comparison with NLTE curve-of-growth results indicates that further systematics may be at work. Sestito \& Randich (2005) also derived Li abundance using the curves of growth of Soderblom et al. (1993a) and the non-LTE corrections of Carlsson et al. (1994), and noted a similar effect of the same order when comparing with measurements for the Hyades (their Table 2). However, considering the previous explanations, an opposite effect would be expected since the Hyades have a super-solar iron abundance.

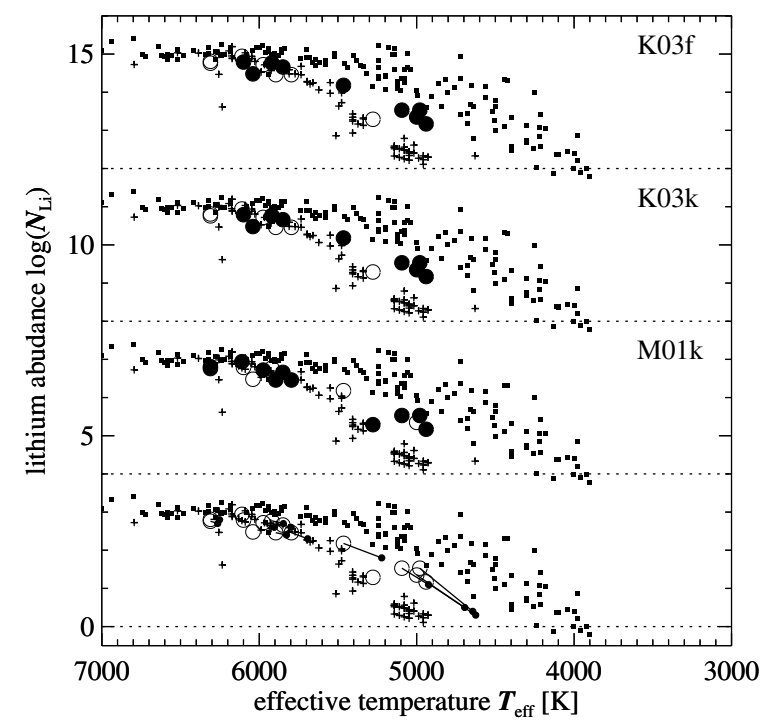

Fig. 6. Effective temperature vs. lithium abundance - The values from Table $6\left(\log N_{\mathrm{NLTE}}^{\mathrm{cog}}(\mathrm{Li})\right)$ are compared to data from King \& Schuler $\left(2005, \log N_{\mathrm{LTE}}^{\mathrm{KTS}}(\mathrm{Li})\right)$ for stars in common, and to the Hyades and Pleiades sequences which are taken from Sestito \& Randich (2005). The layout of the bottom panel follows Fig. 5a, and that of the upper three panels has the same meaning as Fig. 5b. The upper three panels are shifted by arbitrary amounts with respect to the bottom panel. The dotted lines indicate an abundance level of zero to aid the eye.

The strength of the Li absorption and size of the Li abundance are correlated with effective temperature (Figs. 5 and 6). Effective temperatures are obtained from Table 5 if available and otherwise taken from the literature as indicated in Table 6. The spectral type of HD $155674 \mathrm{~A}$ is indicative of an effective temperature of $5250 \mathrm{~K}$ according to Kenyon \& Hartmann (1995), whereas the $\mathrm{H} \alpha$ wings favour a temperature as cool as $4800 \mathrm{~K}$ or even lower.

The UMa group data is compared to the comprehensive compilation of King \& Schuler (2005), who obtained Li equivalent widths and abundances of UMa group members from both the literature and their own measurements (Table 6, Cols. 5 and 9, Figs. 5 and 6). The integrated equivalent widths $W_{\mathrm{Li}}^{\text {int }}$ generally 


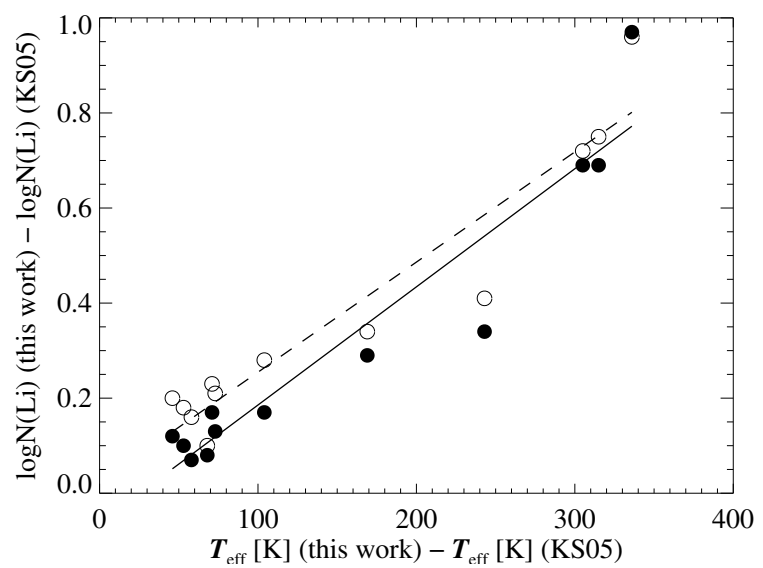

Fig. 7. Systematics of measured Li abundances - for each star in common with King \& Schuler (2005), the difference of measured Li abundance is plotted versus the difference of adopted effective temperatures. The lines represent linear fits to the data. The values of King \& Schuler (2005) given in Col. 9 of Table $6\left(\log N_{\mathrm{LTE}}^{\mathrm{KS} 05}(\mathrm{Li})\right)$ are subtracted from the LTE values presented in Col. $6\left(\log N_{\mathrm{LTE}}^{\mathrm{cog}}(\mathrm{Li})\right.$, filled circles, solid line) and Col. $8\left(\log N_{\mathrm{LTE}}^{\mathrm{fit}}(\mathrm{Li})\right.$, circles, dashed line). They are not compared to those of Col. 7, which were corrected for deviations from LTE and are used later. See also Fig. 3 for the systematic differences in effective temperature.

agree well with those given by King \& Schuler $\left(2005, W_{\mathrm{Li}}^{\mathrm{KS} 05}\right)$. The average difference amounts to $1.6 \pm 7.4 \mathrm{~m} \AA$, which is well within the error bars. Only in the case of HD 109647, the values differ by as much as $21 \mathrm{~m} \AA$. King \& Schuler (2005) adopted a Li equivalent width of $25 \mathrm{~m} \AA$ for this star from Soderblom et al. (1993b). The inspection of Table 1 of the latter shows that it is the only star with substantially conflicting measurements. The given values range from 14 to $40 \mathrm{~m} \AA$, the latter being in good agreement with the present work.

The values of the present work are not only compared to previous measurements of the UMa group. Furthermore, they are studied relative to other stellar groups in a way as consistently as possible. Therefore, the UMa group is compared to the Pleiades and Hyades using data provided by Sestito \& Randich (2005) (Figs. 5 and 6). Some important points have to be kept in mind. Uncertainties in the effective temperature may shift the Li sequence considerably. Except for the coolest stars, the effective temperatures were derived in a very homogeneous way making the UMa sequence very reliable in a relative sense. Figures 5 and 6 reproduce the systematic offset from the effective temperature scale of King \& Schuler (2005), which increases towards cooler stars. One cannot exclude similar offsets from the temperature scale of Sestito \& Randich (2005), who derived effective temperature from $B-V$ colour in a way similar to King \& Schuler (2005).

The derived Li abundance is affected substantially by those temperature offsets. While the equivalent widths presented by King \& Schuler (2005) and this work agree well (Fig. 5), the temperature offsets imply an offset in $\mathrm{Li}$ abundance of corresponding strength. King \& Schuler (2005) derived Li abundances by interpolating in curve-of-growth tables generated by the LTE analysis package MOOG. These values can be compared with the LTE values derived in the present work (Table 6, Cols. 6, 8). Figure 7 shows that differences strongly correlate with the systematics in effective temperature. The effect was modelled qualitatively using the line synthesis tools of the present study. A decrease in effective temperature implies a decrease in $\mathrm{Li}$ abundance when fitting the same spectrum. Both the $\mathrm{Li}$ abundance and the temperature offsets thus conspire in a peculiar way (Fig. 6) so that in the end, the position of the UMa Li sequence remains virtually unchanged with respect to the sequences of the Hyades and Pleiades.

Differences in metallicity are also known to strongly affect the derivation of $\mathrm{Li}$ abundance. While the present work accounts for iron abundance within the synthetic line formation, the Li abundances presented by Sestito \& Randich (2005) are not corrected for metallicity. This particularly concerns the more metal-rich Hyades (cf. Soderblom et al. 1993b; King \& Schuler 2005). The effect of the overall enhanced opacity on the derivation of Li abundance by LTE line formation was estimated by rescaling the $\mathrm{Li}$ abundance of the UMa group member HD 11131 , i.e., changing $[\mathrm{Fe} / \mathrm{H}]$ by +0.23 dex to match the average abundance of Hyades members. To reproduce the Li line of this star, Li abundance had to be changed by -0.23 dex. Despite this significant variation, the position of HD 11131 in Fig. 6 is hardly changed with respect to the Hyades sequence.

The distribution of the warmer UMa group members in Figs. 5 and 6 overlaps with the distributions of the Pleiades and the Hyades. Soderblom et al. (1993b) argue that the scatter of equivalent widths in the UMa group in this temperature range is real. They compare pairs of UMa group members with very similar effective temperatures and conclude that differences in $\mathrm{Li}$ abundance cannot be explained by uncertain effective temperatures. They also note the reduced scatter of equivalent widths in the cooler $\mathrm{K}$ dwarf regime around $5000 \mathrm{~K}$ compared to the Pleiades. This is attributed to the small numbers and supported by the argument that a random selection of the more numerous Pleiades within one temperature bin would also produce a very narrow range of equivalent widths. The increased scatter below $5200 \mathrm{~K}$ noticed by King \& Schuler (2005) is not observed in the present work, which is caused by the small numbers and the way in which the sample was selected.

The cooler UMa group stars clearly have different properties from those in the Hyades and Pleiades, although the plot region around $5500 \mathrm{~K}$ is sparsely populated. In the cool regime, lithium absorption of young stars strongly correlates with the age (e.g., Sestito \& Randich 2005, for a comprehensive study). The Pleiades have an age of roughly $110 \mathrm{Myr}$ (Terndrup et al. 2000), while the Hyades are much older with an age of $\approx 625 \mathrm{Myr}$ (Perryman et al. 1998). In the case of two stars, HD $152863 \mathrm{~A}$ and HD 205435, only upper limits could be measured, which is probably related to their giant status. Although trends in equivalent widths do not necessarily reflect trends in $\mathrm{Li}$ abundance in an unambiguous way, the distribution of the cool UMa group dwarf members can be well distinguished from the Pleiades and the Hyades. This conclusion is generally true for $\mathrm{Li}$ abundance as well, although it is then based on a smaller number of stars. The comparison with the Hyades in the present work and also in King \& Schuler (2005) benefits from the increased number of Hyades members with known Li abundance compared to Soderblom et al. (1993b).

The UMa group sequence turns out extremely sharp between $5000 \mathrm{~K}$ and solar temperatures when considering the kinematic (K03k) and final members (K03f) of K03. The deviant HD 41593 is part of the kinematic member list of M01. Soderblom et al. (1993b) already noted that the Li abundance of this particular star is unusually low compared to the other UMa group members.

These conclusions are based on relatively few stars. However, they gain confidence because only stars with a high membership probability are considered. All unsure candidates 


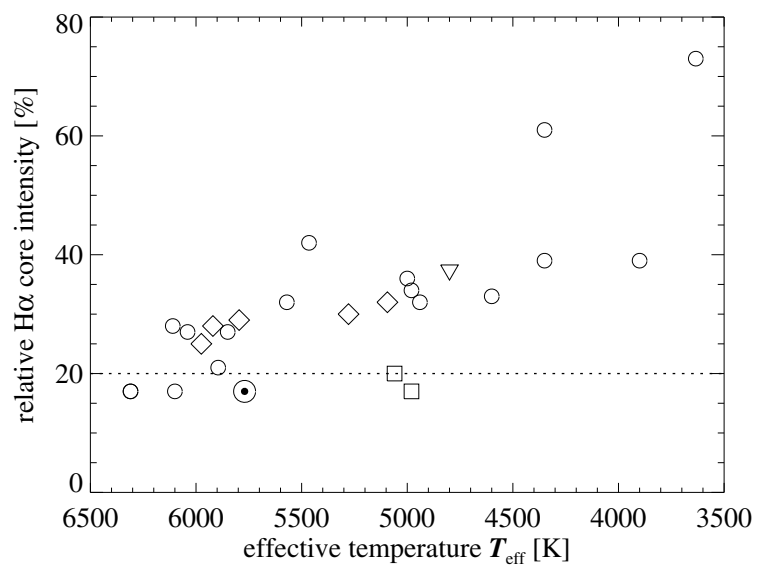

Fig. 8. Effective temperature vs. relative intensity of the $\mathrm{H} \alpha$ core - The UMa group members (Table 6) are compared to the Sun $(\odot)$. Diamonds indicate data taken from Fuhrmann (2004), all other symbols depict values derived in the present work. The squares denote the two giants HD 152863 A and HD 205435. HD 155674 A, which has an uncertain effective temperature, is indicated by the triangle. All Sun-like or cooler dwarf members of the UMa group have a relative intensity of the core of more than $20 \%$ (dashed line).

are excluded from the present work. Thus, lithium equivalent width and abundance seem a valuable membership criterion for UMa group candidates cooler than the Sun. Down to an effective temperature of $\approx 4900 \mathrm{~K}$, the strength of Li absorption as well as $\mathrm{Li}$ abundance should be in-between values for the sequences of the Pleiades and the Hyades. In this temperature range, neither cool giants nor cooler dwarf members should have any measurable Li line.

\subsubsection{Infilling of the $\mathrm{H} \alpha$ core}

Chromospheric activity is usually quantified using indices based on CaII H\&K and the CaII infrared triplet (see Soderblom \& Mayor 1993b; King et al. 2003, for the UMa group). Unfortunately, these lines are not present in the spectra available to the present work. However, it is possible to address the chromospheric infilling of the $\mathrm{H} \alpha$ core. Lyra \& Porto de Mello (2005) argue that there are even some advantages compared to Ca II H\&K.

Fuhrmann (2004) note that the $\mathrm{H} \alpha$ cores of Ursa Major stars are filled-in by about $10 \%$ compared to inactive main-sequence stars of similar effective temperature. The present work allows one to verify this behaviour for a larger sample of UMa group members.

To be consistent with the work of Fuhrmann (2004), the intensity level of the core of the $\mathrm{H} \alpha$ line is assessed (Table 6) and compared to the solar case. The values are simply obtained by taking the minimum flux between 6560 and $6565 \AA$. In the case of HD 11131, HD 26923, HD 39587, HD 41593, and HD 59747, the values given by Fuhrmann (2004, Fig. 16) are adopted.

Figure 8 generally confirms the findings of Fuhrmann (2004). The UMa group dwarf members display an H $\alpha$ core elevated by on average about $10 \%$. A tight sequence extends towards the coolest temperatures and clearly shows an offset with respect to the inactive solar case. The cool deviating objects are the two giants and a few outliers with obviously enhanced chromospheric activity (HD 28495, HD 238224, and HD 95650). The samples M01k, K03k, and K03f (according to Table 3) tend to follow the same behaviour. As for the Li distribution, the identification of any deviating trends is limited by the small numbers of data points.

Figure 8 allows one to conclude that no Sun-like or cooler UMa dwarf member is expected with a relative $\mathrm{H} \alpha$ core intensity of less than about $20 \%$. The definition of an upper limit, however, is found to be far less straightforward since individual members may have a significantly enhanced infilling.

To more reliably compare the chromospheric infilling of UMa group members with that of other stellar groups, in particular the Pleiades and the Hyades, the chromospheric flux is measured according to the method of Lyra \& Porto de Mello (2005). They showed that members of different stellar groups display a different average level of chromospheric flux.

Our measurements, which are produced with the method of Lyra \& Porto de Mello (2005), are shown in Table 6. The normalized spectra are integrated in both the $\mathrm{H} \alpha$ core $\left(W_{\mathrm{H} \alpha}\right)$ with a width of $1.7 \AA$ and Willstrop's band (6550-6600 $⿱$; $\left.W_{50}\right)$. The absolute flux in the $\mathrm{H} \alpha$ core $F_{\mathrm{H} \alpha}$ is calculated from the ratio of $W_{\mathrm{H} \alpha}$ to $W_{50}$, using the absolute flux calibration and the $(V-R)-T_{\text {eff }}$ calibration given by Lyra \& Porto de Mello (2005). Finally, photospheric flux is subtracted from $F_{\mathrm{H} \alpha}$ using the lower envelope in their Fig. $3^{13}$. Negative values close to zero are due to the intrinsic uncertainty on the photospheric flux corrections since the true photosperic flux is not known (for a discussion see Lyra \& Porto de Mello 2005). The calibrations provided by Lyra \& Porto de Mello (2005) are only valid at effective temperatures as low as $5000 \mathrm{~K}$. An extrapolation to the coolest stars in the present sample yields unrealistic results, which are excluded here (HD 95650, HD 155674 B, HD 238087, HD 238224). Figure 9 compares the values obtained in the present work to the values of Pleiades, UMa group, and Hyades members assessed by Lyra \& Porto de Mello (2005). The chromospheric flux of the Sun is measured from the exposures of the lunar disk obtained for the present work. On average, it is $3.58 \pm 0.35$, in excellent agreement with the value given by Lyra \& Porto de Mello (2005) (3.44 \pm 0.34$)$. At solar temperatures, the average flux levels of Pleiades, UMa group, and Hyades can be distinguished well, the Pleiades showing on average the largest and the Hyades having the lowest values.

The bottom panel of Fig. 9 compares the measurements of stars common to both studies. For HD 11131, HD 26923, HD 39587, and HD 41593, Lyra \& Porto de Mello (2005) give the fluxes $12.44,6.72,10.25$, and $9.93\left[10^{5} \mathrm{erg} \mathrm{cm}^{-2} \mathrm{~s}^{-1}\right]$. The average difference from the measurements in the present work is $0.88 \pm 1.51\left[10^{5} \mathrm{erg} \mathrm{cm}^{-2} \mathrm{~s}^{-1}\right]$. On the one hand, this discrepancy is small compared to the total range of possible values covering three orders of magnitude. On the other hand, Pleiades, UMa group, and Hyades display fluxes of the same order of magnitude, so that the slight discrepancies between the present work and that of Lyra \& Porto de Mello (2005) may become crucial.

Soderblom \& Mayor (1993b) assessed a chromospheric emission index of $\mathrm{H} \alpha$ by subtracting the profile of an inactive star and obtaining the equivalent widths. These were converted to chromospheric flux ratios and rescaled to be compared to the index $R_{\mathrm{HK}}^{\prime}$, which is based on the Ca II H\&K lines. These values $\left(R_{\mathrm{HK}}^{\mathrm{H} \alpha}\right)$ are displayed in Fig. 10 for the stars in common with both the present work and Lyra \& Porto de Mello (2005). The work of Soderblom \& Mayor (1993b) has eight stars in common with the present work and six with Lyra \& Porto de Mello (2005), but only four stars are common to all three studies (indicated by solid lines in Fig. 10). The comparison with Lyra \& Porto de Mello (2005) allows for the inclusion of

\footnotetext{
13 The coefficients of the spline were kindly provided by W. Lyra.
} 


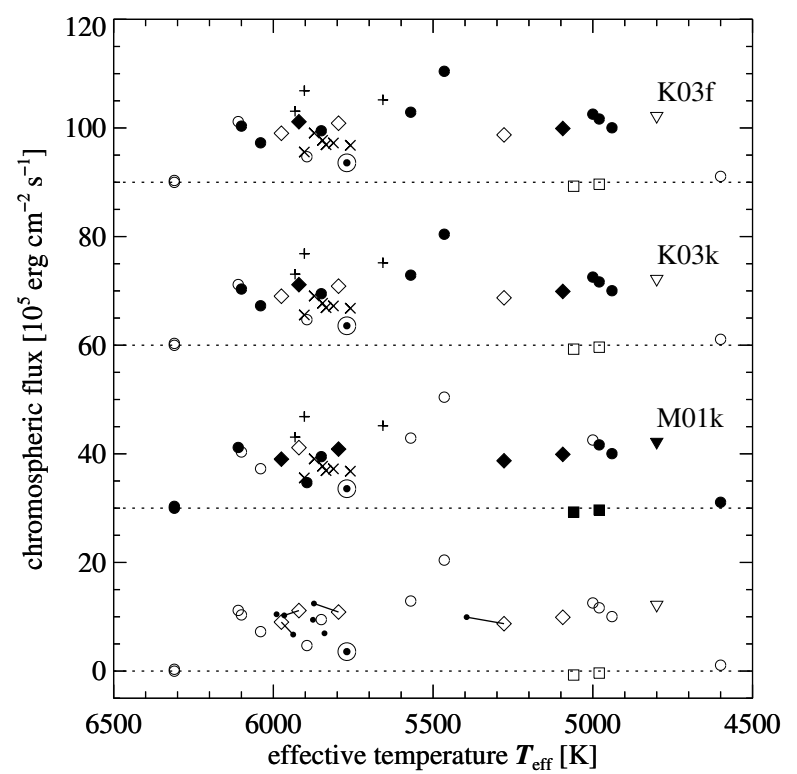

Fig. 9. Effective temperature vs. chromospheric flux $F_{\mathrm{H} \alpha}$ (Lyra \& Porto de Mello 2005) - The layout corresponds to Fig. 6 indicating the three different samples $M 01 k, K 03 k$, and $K 03 f$ by filled symbols in the upper three panels, which are shifted by arbitrary amounts with respect to the bottom panel. The dotted lines indicate a flux level of zero in order to aid the eye. Circles, squares, and diamonds indicate the measurements obtained in the present work. As in Fig. 8, the stars analysed by F04 are depicted by diamonds, the two giants HD $152863 \mathrm{~A}$ and HD 205435 by squares, and HD $155674 \mathrm{~A}$ by the triangle. The measurements are compared to the Sun $(\odot)$ and the measurements of Lyra \& Porto de Mello (2005) for the Pleiades (plus signs), UMa group (dots), and the Hyades (crosses). The solid lines in the lowest panel connect measurements of the present work and Lyra \& Porto de Mello (2005) for stars in common. There, the other data from Lyra \& Porto de Mello (2005) are omitted for clarity.

HD 26913 and HD 147513, which are not studied in the present work.

Some stars in common could not be included. Soderblom \& Mayor (1993b) only determined an upper limit to the value of $R_{\mathrm{HK}}^{\mathrm{H} \alpha}$ for HD 184960, and there is no such measurement at all for HD 38393 and HD 155674 A and B. HD 184960 was designated a probable spectrosopic non-member by Soderblom \& Mayor (1993b) while HD 38393 and HD 155674 A and B are possible spectroscopic members. However, K03 classify HD 38393 and HD 184960 as activity-based non-members, while no activity indicator at all was assessed for HD $155674 \mathrm{~A}$ and B, inferring that they are additional UMa candiates. The present work assigns HD $155674 \mathrm{~A}$ an UMa-like chromospheric emission measure of 11.87, whereas HD 155674 B was excluded from the analysis. HD 38393 and HD 184960 do not exhibit any chromospheric $\mathrm{H} \alpha$ flux. These two objects are the two hottest stars studied here, and low chromospheric emission is possibly common among these warm UMa group members. At these effective temperatures, Lyra \& Porto de Mello (2005) did not study any Hyades stars, so that a more detailed comparison with Soderblom \& Mayor (1993b) and K03 cannot be made here.

It can be noticed from Fig. 10 that the different chromospheric $\mathrm{H} \alpha$ emission measures behave in an expected way. They are correlated although substantial scatter is present. All objects in common that showed UMa-like chromospheric $\mathrm{H} \alpha$ emission in Soderblom \& Mayor (1993b), i.e., chromospheric indices larger than the Hyades mean relation, also have a chromospheric flux measure that is larger than 6.0 in the present work as well

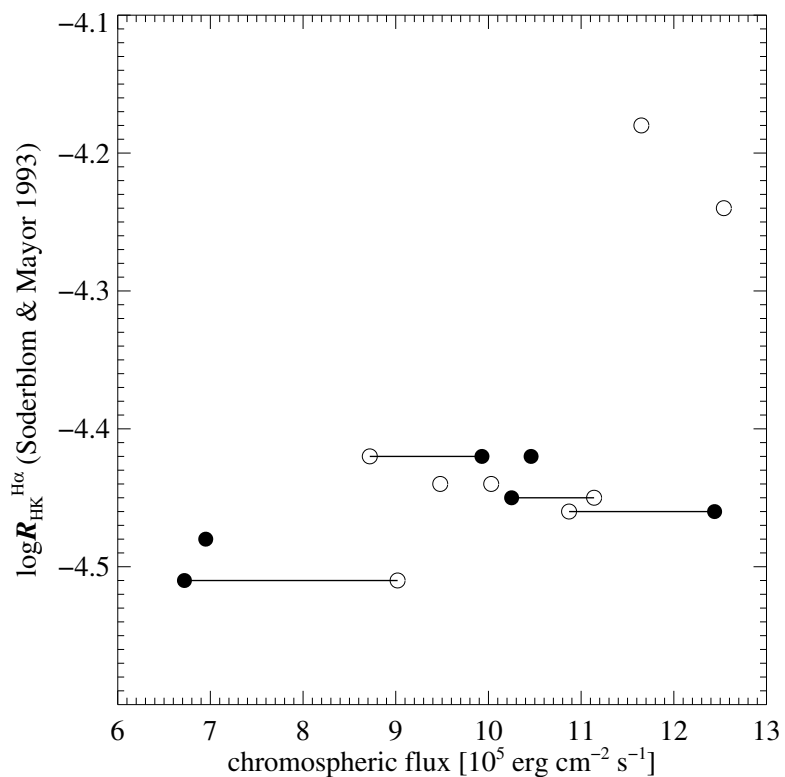

Fig. 10. Chromospheric flux $F_{\mathrm{H} \alpha}$ vs. $R_{\mathrm{HK}}^{\mathrm{H} \alpha}-$ The chromospheric emission index for $\mathrm{H} \alpha$ of Soderblom \& Mayor (1993b) is compared to the chromospheric flux measure according to Lyra \& Porto de Mello (2005) for UMa group stars in common. Empty circles indicate the chromospheric flux measurements of the present work, filled circles those of Lyra \& Porto de Mello (2005). Similar to Fig. 9, the lines connect data points with different chromospheric flux measurements for the same star.

as in Lyra \& Porto de Mello (2005). This flux level separates the young and the old dwarf stars in Lyra \& Porto de Mello (2005, their Fig. 10). Soderblom \& Mayor (1993a, Fig. 2) compared the $\mathrm{H} \alpha$ emission of UMa group members to the Pleiades. The hotter UMa group stars overlap with the lower boundary of the wide distribution of the Pleiades. This overlap is also generally found in the present work and the work of Lyra \& Porto de Mello (2005), although represented by only three Pleiades stars.

In contrast to Lyra \& Porto de Mello (2005), the present work allows one to study the dependence of the chromospheric flux for a large range of effective temperature, i.e., stellar mass. Noticable flux levels are only measured at temperatures of $6000 \mathrm{~K}$ and below. At solar temperatures, a dependence on age is clearly noticed (Lyra \& Porto de Mello 2005). The two UMa group giants HD 152863 A and HD 205435 exhibit a significantly lower chromospheric flux level compared to dwarf stars of similar temperature.

In the temperature range from $6000 \mathrm{~K}$ down to $5000 \mathrm{~K}$, the difference between Pleiades, UMa group, and Hyades is no longer so clear as in the case of only the solar temperature dwarfs. The $K 03 f$ sample displays chromospheric emission at the level of the Hyades or larger, in agreement with the spectroscopic criteria applied by Soderblom \& Mayor (1993b) and K03. If only the dwarf stars cooler than $\approx 6000 \mathrm{~K}$ are considered, then the chromospheric emission of all three samples M01k, K03k, and $K 03 f$ is similar to the Hyades or stronger. The only exception is HD 24916 A, the coolest star with chromospheric emission measured in the present work. The spectroscopic criterion should thus be restricted to stars hotter than $5000 \mathrm{~K}$ (on the effective temperature scale of the present work).

\section{Summary and conclusions}

Stellar parameters of 11 Ursa Major (UMa) group members have been derived homogeneously from spectra of both high 
resolving power and high signal-to-noise ratio using the methods of Fuhrmann (2004, and preceeding work). The method was only partially successful in the case of four stars so that surface gravities had to be calculated from the Hipparcos distance.

The consistency of the analysis was proven by reproducing data for the Sun and a possible UMa group member already analysed by Fuhrmann (2004), and by comparing the spectroscopic distance with the geometric Hipparcos distance. Furthermore, the derived values were found to be in good agreement with literature values, although some systematics were found relative to King \& Schuler (2005), who also analysed a larger sample of UMa group members.

To study the sample of UMa group members as a whole, the data set was complemented with data for 6 more UMa group members analysed with the same methods by Fuhrmann (2004) and König et al. (2006). An iron abundance of $[\mathrm{Fe} / \mathrm{H}]=-0.03 \pm 0.05$ was obtained for the UMa group, which is about 0.06 dex higher than previous estimates. The $[\mathrm{Fe} / \mathrm{H}]$ abundance is thus indistinguishable from that of the Pleiades but clearly different from that of the Hyades. The iron and magnesium abundances of the UMa group members correspond to those of the thin disk population.

The equivalent widths of the Li I $6708 \AA$ feature were measured for in total 25 UMa group members and Li abundance for 16 of these stars. At effective temperatures lower than solar, the strength of the Li line and the Li abundance can be clearly distinguished from that of the Pleiades and the Hyades.

Chromospheric emission was considered by two different approaches. The flux level of the $\mathrm{H} \alpha$ core of 25 objects was measured according to Fuhrmann (2004). In the case of the Sun-like and cooler UMa group members, it is elevated by about $10 \%$ with respect to the Sun, a result which is in line with previous findings by Fuhrmann (2004). While the lower boundary of chromospheric infilling seems clearly defined, substantially higher values were found for some individual stars. Furthermore, chromospheric flux is calculated according to Lyra \& Porto de Mello (2005) for 21 sample stars hotter than $5000 \mathrm{~K}$. While the emission level is weaker than that of the Pleiades and stronger than that of the Hyades at solar temperatures, the distributions partly overlap at cooler temperatures. The chromospheric flux of all UMa group dwarfs between effective temperatures of $5000 \mathrm{~K}$ and $6000 \mathrm{~K}$ remains similar to that of the Hyades or is higher.

In previous works, it was found to be rather difficult to establish a definite list of bona-fide Ursa Major group members. Therefore, a simplistic approach was chosen in the present work by not trying to create a single and definite list that would be the basis of the definition of spectroscopic membership criteria. Different lists from the literature were adopted instead by considering the distribution of iron abundance, Li absorption and $\mathrm{H} \alpha$ filling-in separately for each of these lists. A qualitative difference of the results of the samples cannot be assessed robustly since the number of stars studied is small. The kinematic and final members of King et al. (2003) form remarkably tight sequences of $\mathrm{Li}$ equivalent width and abundance and a fairly homogeneous level of the chromospheric $\mathrm{H} \alpha$ emission measure.

The purely kinematically selected stars appear similar in terms of their spectroscopic properties. In particular, the spread of iron abundance is much smaller than the total spread for the thin disk population. Most notably, the scatter of iron abundance is of the same order as the typical abundance measurement error bar. Similarly, the scatter of Li abundance is small along a temperature-dependent sequence and clearly differs from that of other groups of stars, supporting a young age for the UMa group in-between that for the Pleiades and the Hyades. Chromospheric emission in the $\mathrm{H} \alpha$ line core is also indicative of youth since it is similar to the Hyades. Thus, the present study corroborates previous findings that the existence of the UMa group does not depend entirly on unsure kinematic criteria alone. The present work suggests that spectroscopic criteria may help to identify additional UMa group members.

Membership criteria derived from the mean iron abundance, the $\mathrm{Li}$ absorption, and the $\mathrm{H} \alpha$ infilling can be used to exclude non-members (King et al. 2003), i.e., the fulfillment of these criteria is necessary but insufficient for deciding on membership. UMa group members should have an iron abundance $[\mathrm{Fe} / \mathrm{H}]$ in the range $[-0.14,0.06]$. Members cooler than the Sun and hotter than $5300 \mathrm{~K}$ are expected to have stronger Li absorption than the Hyades but weaker than $100 \mathrm{~m} \AA$. The Li feature should be present in the range $5300-4900 \mathrm{~K}$ but weaker than $50 \mathrm{~m} \AA$. Giants and cooler dwarfs in the UMa group are not expected to have any measurable Li I $6708 \AA$ feature. The flux level in the $\mathrm{H} \alpha$ core at effective temperatures between $5000 \mathrm{~K}$ and $6000 \mathrm{~K}$ is similar to that of the Hyades or higher. There is no measurable infilling for the hotter stars and the giants. Chromospheric emission may reach extreme levels in the case of the coolest UMa group members.

These criteria may help us to exclude non-members but cannot be used as a membership criterion in a positive sense. For this purpose, a complete census of stellar kinematic groups in the solar neighbourhood has to be assessed. To enable an accurate comparison, possible systematics have to be removed, which may in turn originate in inhomogeneous data sets.

The presented spectroscopic criteria may certainly help us to identify possible members of the UMa group among the kinematic candidates. For this purpose, new candidates have to be analysed spectroscopically in the same way as in the present work or at least in a very similar way. Then, chemical abundance, in particular $\mathrm{Fe}$ and $\mathrm{Li}$, as well as chromospheric infilling can be addressed by the spectroscopic criteria in a very uniform way.

The reader may have noticed that the spectroscopic criteria are based on only very few kinematic members, and will have to be placed on firmer grounds. Therefore, the first challenge will be to follow Soderblom \& Mayor (1993b), King et al. (2003), and Fuhrmann (2004) in studying whether new spectroscopic UMa group candidates also cluster tightly in kinematic space. In this way, the kinematic membership criteria can be refined and more stars included in the definition of spectroscopic criteria. A larger list of members can then be established in a more reliable way.

Acknowledgements. M.A. wants to thank Ralph Neuhäuser and Gregor. E. Morfill who supervised and supported the Ph.D. Thesis. M.A. is indebted to Klaus Fuhrmann who kindly provided some of his FOCES spectra and his analysis package and gives ongoing support. Furthermore, M.A. likes to thank Brigitte König for help in the analysis of young stars and Andreas Korn for software to add spectra. Moreover, M.A. gratefully acknowledges fruitful discussions with Giancarlo Pace, Günter Wuchterl, and Marc Hempel. M.A. and E.G. thank the support staff at the observatories at Calar Alto and at Tautenburg. We thank the anonymous referee for comments and suggestions which clearly helped to improve this work. M.A. was supported by a graduate scholarship of the Cusanuswerk, one of the national student elite programs of Germany and by a scholarship (reference SFRH/BPD/26817/2006) granted by the Fundação para a Ciência e a Tecnologia (FCT), Portugal. M.A. acknowledges research funding granted by the Deutsche Forschungsgemeinschaft (DFG) under the projects NE 515/16 and 17, and RE 1664/4-1. This research has made use of the extract stellar request type of the Vienna Atomic Line Database (VALD), the SIMBAD database, operated at CDS, Strasbourg, France, and NASA's Astrophysics Data System Bibliographic Services. 
Appendix A: List of Fe lines used in this work

Table A.1. Data of Fe I and II lines used in this work.

\begin{tabular}{|c|c|c|c|c|c|c|c|c|c|c|c|}
\hline$\lambda[\AA]$ & Ion. stage & $\chi_{\text {low }}[\mathrm{eV}]$ & $\log g f$ & $\log C_{6}$ & $\gamma_{\mathrm{rad}}\left[10^{8} 1 / \mathrm{s}\right]$ & $\lambda[\AA]$ & Ion. stage & $\chi_{\text {low }}[\mathrm{eV}]$ & $\log g f$ & $\log C_{6}$ & $\gamma_{\mathrm{rad}}\left[10^{8} 1 / \mathrm{s}\right]$ \\
\hline 4993.35 & 2 & 2.80 & -3.73 & -31.60 & 3.0500 & 6252.57 & 1 & 2.40 & -1.56 & -31.92 & 0.87000 \\
\hline 4994.14 & 1 & 0.91 & -3.01 & -31.97 & 0.15000 & 6297.80 & 1 & 2.22 & -2.71 & -31.86 & 1.5500 \\
\hline 5127.37 & 1 & 0.91 & -3.20 & -31.97 & 0.16600 & 6301.51 & 1 & 3.65 & -0.67 & -30.70 & 0.80500 \\
\hline 5197.58 & 2 & 3.23 & -2.31 & -31.80 & 3.0300 & 6330.85 & 1 & 4.73 & -1.15 & -31.69 & 2.3840 \\
\hline 5198.72 & 1 & 2.22 & -2.12 & -31.50 & 1.6100 & 6335.34 & 1 & 2.20 & -2.22 & -31.86 & 1.6600 \\
\hline 5217.40 & 1 & 3.21 & -1.08 & -30.60 & 0.76000 & 6336.83 & 1 & 3.69 & -0.80 & -30.70 & 0.81100 \\
\hline 5223.19 & 1 & 3.64 & -2.25 & -31.91 & 0.78600 & 6369.46 & 2 & 2.89 & -4.19 & -31.50 & 2.9000 \\
\hline 5234.63 & 2 & 3.22 & -2.23 & -31.80 & 3.0700 & 6380.75 & 1 & 4.19 & -1.24 & -31.70 & 0.73503 \\
\hline 5242.50 & 1 & 3.63 & -0.87 & -31.70 & 0.57500 & 6393.61 & 1 & 2.43 & -1.39 & -31.92 & 0.93200 \\
\hline 5264.81 & 2 & 3.23 & -3.11 & -32.19 & 4.1100 & 6411.66 & 1 & 3.65 & -0.56 & -30.70 & 0.80300 \\
\hline 5281.80 & 1 & 3.04 & -0.91 & -30.90 & 1.0200 & 6416.93 & 2 & 3.89 & -2.67 & -31.90 & 3.3700 \\
\hline 5284.11 & 2 & 2.89 & -3.12 & -32.11 & 3.4300 & 6430.85 & 1 & 2.18 & -2.02 & -31.86 & 1.6500 \\
\hline 5295.32 & 1 & 4.42 & -1.52 & -31.15 & 1.8380 & 6432.68 & 2 & 2.89 & -3.63 & -31.50 & 2.9000 \\
\hline 5302.31 & 1 & 3.28 & -0.75 & -30.60 & 0.74900 & 6436.41 & 1 & 4.19 & -2.39 & -31.96 & 0.30403 \\
\hline 5325.56 & 2 & 3.22 & -3.22 & -31.80 & 3.1000 & 6456.39 & 2 & 3.90 & -2.09 & -31.90 & 3.3700 \\
\hline 5339.94 & 1 & 3.27 & -0.65 & -30.60 & 0.74900 & 6481.88 & 1 & 2.28 & -2.90 & -31.91 & 1.5500 \\
\hline 5364.88 & 1 & 4.45 & 0.39 & -31.15 & 1.8550 & 6498.94 & 1 & 0.96 & -4.62 & -32.02 & 0.000435 \\
\hline 5367.48 & 1 & 4.42 & 0.49 & -31.15 & 1.8500 & 6516.09 & 2 & 2.89 & -3.34 & -31.50 & 2.9100 \\
\hline 5379.58 & 1 & 3.69 & -1.44 & -31.70 & 0.57500 & 6591.32 & 1 & 4.59 & -2.01 & -31.80 & 1.4000 \\
\hline 5393.17 & 1 & 3.24 & -0.74 & -30.60 & 0.74800 & 6593.88 & 1 & 2.43 & -2.29 & -31.92 & 0.86200 \\
\hline 5414.07 & 2 & 3.22 & -3.61 & -32.19 & 4.1200 & 6608.03 & 1 & 2.28 & -3.99 & -31.91 & 1.6600 \\
\hline 5425.25 & 2 & 3.20 & -3.28 & -31.80 & 2.9900 & 6627.55 & 1 & 4.55 & -1.48 & -31.44 & 1.7530 \\
\hline 5497.52 & 1 & 1.01 & -2.82 & -31.99 & 0.14200 & 6704.48 & 1 & 4.22 & -2.62 & -31.16 & - \\
\hline 5522.45 & 1 & 4.21 & -1.40 & -30.80 & 0.89800 & 6710.32 & 1 & 1.48 & -4.84 & -31.97 & 0.16600 \\
\hline 5534.85 & 2 & 3.24 & -2.83 & -32.18 & 2.9900 & 6713.75 & 1 & 4.80 & -1.42 & -31.30 & 2.3550 \\
\hline 5546.51 & 1 & 4.37 & -1.05 & -31.20 & 1.7860 & 6725.36 & 1 & 4.10 & -2.21 & -31.37 & 2.0910 \\
\hline 5560.22 & 1 & 4.43 & -0.96 & -31.50 & 1.6410 & 6733.15 & 1 & 4.64 & -1.43 & -31.37 & 2.2680 \\
\hline 5576.10 & 1 & 3.43 & -0.79 & -30.85 & 0.75300 & 6750.16 & 1 & 2.42 & -2.53 & -31.95 & 0.0770 \\
\hline 5611.36 & 1 & 3.64 & -2.93 & -31.91 & 1.2500 & 6786.87 & 1 & 4.19 & -1.90 & -31.36 & 1.9860 \\
\hline 5618.64 & 1 & 4.21 & -1.22 & -31.46 & 1.0450 & 6806.85 & 1 & 2.73 & -3.11 & -31.83 & 1.0200 \\
\hline 5636.70 & 1 & 3.64 & -2.52 & -31.94 & 0.39000 & 6810.27 & 1 & 4.61 & -0.94 & -30.90 & 2.3010 \\
\hline 5638.27 & 1 & 4.22 & -0.65 & -31.00 & 1.9430 & 6820.37 & 1 & 4.64 & -1.11 & -30.90 & 2.2200 \\
\hline 5651.47 & 1 & 4.47 & -1.77 & -31.30 & 1.6310 & 6837.01 & 1 & 4.59 & -1.74 & -31.81 & 0.52405 \\
\hline 5679.03 & 1 & 4.65 & -0.64 & -30.80 & 1.3990 & 6843.66 & 1 & 4.55 & -0.73 & -31.10 & 1.9230 \\
\hline 5741.85 & 1 & 4.26 & -1.63 & -31.10 & 2.1080 & 6857.25 & 1 & 4.08 & -2.08 & -31.40 & 0.25303 \\
\hline 5855.08 & 1 & 4.61 & -1.54 & -31.75 & 1.9110 & 6862.50 & 1 & 4.56 & -1.43 & -31.30 & 3.5400 \\
\hline 5856.09 & 1 & 4.29 & -1.55 & -31.91 & 0.86200 & 6936.50 & 1 & 4.61 & -2.20 & -31.69 & 3.2000 \\
\hline 5862.36 & 1 & 4.55 & -0.16 & -31.30 & 1.8970 & 6945.21 & 1 & 2.42 & -2.39 & -31.95 & 0.15700 \\
\hline 5930.19 & 1 & 4.65 & -0.02 & -31.30 & 1.8530 & 6978.86 & 1 & 2.48 & -2.39 & -31.95 & 0.07700 \\
\hline 5956.70 & 1 & 0.86 & -4.54 & -32.01 & 0.000271 & 7219.69 & 1 & 4.08 & -1.49 & -31.91 & 1.6300 \\
\hline 5991.38 & 2 & 3.15 & -3.59 & -31.60 & 3.4600 & 7222.40 & 2 & 3.89 & -3.26 & -31.60 & 4.1400 \\
\hline 6027.06 & 1 & 4.08 & -1.02 & -31.91 & 0.88503 & 7224.48 & 2 & 3.89 & -3.24 & -31.60 & 4.1400 \\
\hline 6056.01 & 1 & 4.73 & -0.31 & -30.80 & 1.8480 & 7301.57 & 2 & 3.89 & -3.69 & -32.18 & 3.0700 \\
\hline 6065.49 & 1 & 2.61 & -1.38 & -31.92 & 1.0200 & 7306.57 & 1 & 4.18 & -1.54 & -30.80 & 1.6710 \\
\hline 6084.11 & 2 & 3.20 & -3.84 & -31.60 & 3.4300 & 7401.69 & 1 & 4.19 & -1.52 & -31.90 & 0.70303 \\
\hline 6093.65 & 1 & 4.61 & -1.32 & -31.75 & 1.9350 & 7411.16 & 1 & 4.28 & -0.32 & -30.80 & 1.7020 \\
\hline 6096.67 & 1 & 3.98 & -1.79 & -31.10 & 0.45250 & 7421.56 & 1 & 4.64 & -1.70 & -31.82 & 2.5010 \\
\hline 6113.32 & 2 & 3.22 & -4.16 & -31.60 & 3.4100 & 7445.76 & 1 & 4.26 & -0.10 & -30.80 & 1.6990 \\
\hline 6149.25 & 2 & 3.89 & -2.75 & -31.90 & 3.3900 & 7454.00 & 1 & 4.19 & -2.34 & -31.90 & 1.4500 \\
\hline 6151.62 & 1 & 2.18 & -3.29 & -31.86 & 1.5500 & 7479.70 & 2 & 3.89 & -3.70 & -32.18 & 3.1000 \\
\hline 6157.73 & 1 & 4.08 & -1.10 & -31.70 & 0.50203 & 7491.66 & 1 & 4.30 & -0.95 & -30.80 & 1.6920 \\
\hline 6173.34 & 1 & 2.22 & -2.84 & -31.86 & 1.6700 & 7495.08 & 1 & 4.22 & 0.04 & -30.80 & 1.6810 \\
\hline 6200.32 & 1 & 2.61 & -2.31 & -31.92 & 1.0300 & 7498.53 & 1 & 4.14 & -2.16 & -31.90 & 1.1600 \\
\hline 6213.44 & 1 & 2.22 & -2.51 & -31.86 & 1.6500 & 7515.84 & 2 & 3.90 & -3.45 & -31.60 & 4.0900 \\
\hline 6226.74 & 1 & 3.88 & -2.11 & -31.10 & 0.54140 & 7540.44 & 1 & 2.72 & -3.81 & -31.85 & 0.93200 \\
\hline 6232.65 & 1 & 3.65 & -1.20 & -30.70 & 0.80600 & 7568.91 & 1 & 4.28 & -0.82 & -30.80 & 1.6990 \\
\hline 6239.94 & 2 & 3.89 & -3.50 & -31.90 & 3.3800 & 7583.80 & 1 & 3.02 & -1.80 & -31.80 & 1.0200 \\
\hline 6240.65 & 1 & 2.22 & -3.27 & -31.95 & 0.15700 & 7711.73 & 2 & 3.90 & -2.56 & -31.60 & 4.1200 \\
\hline 6246.33 & 1 & 3.60 & -0.73 & -30.70 & 0.79600 & 7748.28 & 1 & 2.95 & -1.56 & -31.80 & 1.0200 \\
\hline 6247.56 & 2 & 3.89 & -2.34 & -31.90 & 3.3800 & 7844.56 & 1 & 4.83 & -1.73 & -31.59 & 2.3380 \\
\hline
\end{tabular}

(1) Central wavelength, (2) ionization stage, (3) lower excitation potential, (4) oscillator strength, (5) van der Waals damping constant, (6) radiative damping constant. The effect of quadratic Stark broadening $\left(\log C_{4}\right)$ on weak iron lines is negligible so that in this work, it is not taken into account for these lines. The radiation damping constant $\left(\gamma_{\mathrm{rad}}\right)$ is calculated with the classical formula in case it is missing in the table. 


\section{References}

Adelman, S. J., Coursey, B. C., \& Harris, E. A. 2000, Inform. Bull. Var. Stars, 5003, 1

Allende Prieto, C., \& Lambert, D. L. 1999, A\&A, 352, 555

Allende Prieto, C., Barklem, P. S., Lambert, D. L., \& Cunha, K. 2004, A\&A, 420, 183

Alonso, A., Arribas, S., \& Martínez-Roger, C. 1995, A\&A, 297, 197

Alonso, A., Arribas, S., \& Martínez-Roger, C. 1996, A\&AS, 117, 227

Ammler, M. 2006a, Characterisation of young nearby stars - The Ursa Major group (Berlin: Der VerkanntenVerlag)

Ammler, M. 2006b, Ph.D. Thesis, university of Jena, Germany, http://www . $\mathrm{db}$-thueringen. de/servlets/DocumentServlet?id=7006

Ammler, M., Guenther, E. W., König, B., \& Neuhäuser, R. 2005, in Proceedings of The 13th Cambridge Workshop on Cool Stars, Stellar Systems and the Sun, ed. F. Favata, G. Hussain, \& B. Battrick, ESA, 1, 391

An, D., Terndrup, D. M., Pinsonneault, M. H., et al. 2007, ApJ, 655, 233

Asiain, R., Figueras, F., Torra, J., \& Chen, B. 1999a, A\&A, 341, 427

Asiain, R., Figueras, F., Torra, J., \& Chen, B. 1999b, A\&A, 344, 376

Balachandran, S. 1990, ApJ, 354, 310

Baraffe, I., Chabrier, G., Allard, F., \& Hauschildt, P. 1998, A\&A, 337, 403

Bessell, M. S. 2000, PASP, 112, 961

Boesgaard, A. M. 1989, ApJ, 336, 798

Boesgaard, A. M., \& Budge, K. G. 1988, ApJ, 332, 410

Boesgaard, A. M., \& Friel, E. D. 1990, ApJ, 351, 467

Boesgaard, A. M., Budge, K. G., \& Burck, E. E. 1988, ApJ, 325, 749

Carlsson, M., Rutten, R. J., Bruls, J. H. M. J., \& Shchukina, N. G. 1994, A\&A, 288,860

Castro, S., Porto de Mello, G. F., \& da Silva, L. 1999, MNRAS, 305, 693

Cayrel, R., Cayrel de Strobel, G., \& Campbell, B. 1985, A\&A, 146, 249

Cayrel, R., Cayrel de Strobel, G., \& Campbell, B. 1988, in The Impact of Very High S/N Spectroscopy on Stellar Physics, IAU Symp., 132, 449

Cayrel de Strobel, G., Soubiran, C., \& Ralite, N. 2001, A\&A, 373, 159

Chen, Y. Q., Nissen, P. E., Zhao, G., Zhang, H. W., \& Benoni, T. 2000, A\&AS, 141,491

Chereul, E., Crézé, M., \& Bienaymé, O. 1999, A\&AS, 135, 5

Chupina, N. V., Reva, V. G., \& Vereshchagin, S. V. 2001, A\&A, 371, 115

de Laverny, P., do Nascimento, J. D., Lèbre, A., \& De Medeiros, J. R. 2003, A\&A, 410, 937

Duncan, D. K. 1981, ApJ, 248, 651

Dziewulski, W. 1916, Bull. Int. Acad. Sci. Cracovie, A, 251

Edvardsson, B., Andersen, J., Gustafsson, B., et al. 1993, A\&A, 275, 101

Eggen, O. J. 1958, MNRAS, 118, 65

Eggen, O. J. 1992, AJ, 104, 1493

Eggen, O. J. 1994, in Galactic and Solar System Optical Astrometry, 191

Eggen, O. J. 1995, AJ, 110, 2862

ESA 1997, VizieR Online Data Catalog, 1239, 0

Fabricius, C., \& Makarov, V. V. 2000, A\&A, 356, 141

Friel, E. D., \& Boesgaard, A. M. 1992, ApJ, 387, 170

Fuhrmann, K. 1993, Ph.D. Thesis, LMU München

Fuhrmann, K. 1998, A\&A, 338, 161

Fuhrmann, K. 2000, http://www.ing.iac.es/ klaus/pap_100.ps

Fuhrmann, K. 2004, Astron. Nachr., 325, 3 (F04)

Fuhrmann, K., Axer, M., \& Gehren, T. 1993, A\&A, 271, 451

Fuhrmann, K., Axer, M., \& Gehren, T. 1994, A\&A, 285, 585

Fuhrmann, K., Pfeiffer, M., Frank, C., Reetz, J., \& Gehren, T. 1997a, A\&A, 323, 909

Fuhrmann, K., Pfeiffer, M. J., \& Bernkopf, J. 1997b, A\&A, 326, 1081

Gaidos, E. J., \& Gonzalez, G. 2002, New Astron., 7, 211

Giannuzzi, M. A. 1979, A\&A, 77, 214

Gratton, R. G., Carretta, E., \& Castelli, F. 1996, A\&A, 314, 191

Gray, D. F. 1984, ApJ, 281, 719

Gray, R. O., Corbally, C. J., Garrison, R. F., McFadden, M. T., \& Robinson, P. E. 2003, AJ, 126, 2048

Grupp, F. 2003, A\&A, 412, 897

Grupp, F. 2004, Ph.D. Thesis, LMU München

Hatzes, A. P., Guenther, E. W., Endl, M., et al. 2005, A\&A, 437, 743

Heintz, W. D. 1988, A\&AS, 72, 543

Herbig, G. H. 1985, ApJ, 289, 269

Hertzsprung, E. 1909, ApJ, 30, 135
Holweger, H. 1979, in Les Élements et leurs Isotopes dans l’Univers, 22nd Liège Symp., Liège

Horne, K. 1986, PASP, 98, 609

Huggins, W. 1871, Roy. Soc. Lond. Proc. Ser. I, 20, 379

Hünsch, M., Randich, S., Hempel, M., Weidner, C., \& Schmitt, J. H. M. M. 2004, A\&A, 418, 539

Kenyon, S., \& Hartmann, L. 1995, ApJS, 101, 117

King, J. R., \& Schuler, S. C. 2005, PASP, 117, 911

King, J., Villarreal, A., Soderblom, D., Gulliver, A., \& Adelman, S. 2003, AJ, 125,1980 (K03)

King, J. R., Soderblom, D. R., Fischer, D., \& Jones, B. F. 2000, ApJ, 533, 944

König, B. 2003, Ph.D. Thesis, LMU Munich

König, B., Fuhrmann, K., Neuhäuser, R., Charbonneau, D., \& Jayawardhana, R. 2002, A\&A, 394, L43

König, B., Guenther, E. W., Esposito, M., \& Hatzes, A. 2006, MNRAS, 365, 1050

Korn, A. J. 2002, in Scientific Drivers for ESO Future VLT/VLTI Instrumentation Proceedings of the ESO Workshop held in Garching, Germany, 11-15 June, 2001, 199

Korn, A. J., Maraston, C., \& Thomas, D. 2005, A\&A, 438, 685

Kupka, F., Piskunov, N., Ryabchikova, T. A., Stempels, H. C., \& Weiss, W. W. 1999, A\&AS, 138, 119

Kupka, F. G., Ryabchikova, T. A., Piskunov, N. E., Stempels, H. C., \& Weiss, W. W. 2000, Balt. Astron., 9, 590

Kurucz, R. 1995, in Highlights of Astronomy, ed. I. Appenzeller, 10, 407

Kurucz, R. L., Furenlid, I., \& Brault, J. T. L. 1984, Solar flux atlas from 296 to $1300 \mathrm{~nm}$, National Solar Observatory Atlas, Sunspot (New Mexico: National Solar Observatory)

Lowrance, P. J., Becklin, E. E., Schneider, G., et al. 2005, AJ, 130, 1845

Luck, R. E., \& Heiter, U. 2005, AJ, 129, 1063

Lyra, W., \& Porto de Mello, G. F. 2005, A\&A, 431, 329

Makarov, V. V., \& Kaplan, G. H. 2005, AJ, 129, 2420

Mallik, S. V. 1998, A\&A, 338, 623

Martin, E. L., \& Montes, D. 1997, A\&A, 318, 805

McLean, I. 1997, Electronic imaging in astronomy - Detectors and Instrumentation, Wiley-Praxis Series in Astronomy and Astrophysics (John Wiley \& Sons Ltd in association with Praxis Publishing Ltd)

McWilliam, A. 1990, ApJS, 74, 1075

Montes, D., López-Santiago, J., Fernández-Figueroa, M. J., \& Gálvez, M. C. 2001a, A\&A, 379, 976

Montes, D., López-Santiago, J., Gálvez, M. C., et al. 2001b, MNRAS, 328, 45 (M01)

Paulson, D. B., Sneden, C., \& Cochran, W. D. 2003, AJ, 125, 3185

Perryman, M. A. C., Lindegren, L., Kovalevsky, J., et al. 1997, A\&A, 323, L49

Perryman, M. A. C., Brown, A. G. A., Lebreton, Y., et al. 1998, A\&A, 331, 81

Pfeiffer, M. J., Frank, C., Baumueller, D., Fuhrmann, K., \& Gehren, T. 1998, A\&AS, 130, 381

Piskunov, N. E., Kupka, F., Ryabchikova, T. A., Weiss, W. W., \& Jeffery, C. S. 1995, A\&AS, 112, 525

Proctor, R. A. 1869, Roy. Soc. Lond. Proc. Ser. I, 18, 169

Reile, C. 1987, Master's Thesis, LMU München

Roman, N. G. 1949, ApJ, 110, 205

Ryabchikova, T. A., Piskunov, N. E., Kupka, F., \& Weiss, W. W. 1997, Balt. Astron., 6, 244

Schmidt-Kaler, T. 1982, in Landolt-Börnstein, ed. K. Schaifers, \& H. Voigt, 2 (Berlin: Springer), 449

Sestito, P., \& Randich, S. 2005, A\&A, 442, 615

Soderblom, D. R., \& Mayor, M. 1993a, ApJ, 402, L5

Soderblom, D. R., \& Mayor, M. 1993b, AJ, 105, 226

Soderblom, D. R., Jones, B. F., Balachandran, S., et al. 1993a, AJ, 106, 1059

Soderblom, D. R., Pilachowski, C. A., Fedele, S. B., \& Jones, B. F. 1993b, AJ, 105,2299

Steinhauer, A. 2003, Ph.D. Thesis

Terndrup, D. M., Stauffer, J. R., Pinsonneault, M. H., et al. 2000, AJ, 119, 1303

Thorburn, J., Hobbs, L., Deliyannis, C., \& Pinsonneault, M. 1993, ApJ, 415, 150

VandenBerg, D. A., Swenson, F. J., Rogers, F. J., Iglesias, C. A., \& Alexander, D. R. 2000, ApJ, 532, 430

von Hoerner, S. 1957, Z. Astrophys., 42, 273

Wielen, R. 1971, A\&A, 13, 309

Wielen, R. 1978, BAAS, 10, 408

Yi, S., Demarque, P., Kim, Y.-C., et al. 2001, ApJS, 136, 417 